\title{
Complementability and maximality in different contexts: ergodic theory, Brownian and poly-adic filtrations
}

\author{
Christophe Leuridan
}

September 24, 2018

\begin{abstract}
The notions of complementability and maximality were introduced in 1974 by Ornstein and Weiss in the context of the automorphisms of a probability space, in 2008 by Brossard and Leuridan in the context of the Brownian filtrations, and in 2017 by Leuridan in the context of the poly-adic filtrations indexed by the nonpositive integers. We present here some striking analogies and also some differences existing between these three contexts.
\end{abstract}

Mathematics Subject Classification: 37A05, 60J05.

Keywords: Automorphisms of Lebesgue spaces, factors, entropy, filtrations indexed by the non-positive integers, poly-adic filtrations, Brownian filtrations, immersed filtrations, complementability, maximality, exchange property.

\section{Introduction}

\section{$1.1 \quad$ General context}

In the present paper, we will work with three types of objects: automorphisms of Lebesgue spaces, Brownian filtrations and filtrations indexed by $\mathbf{Z}$ or $\mathbf{Z}_{-}$; the reason for choosing $\mathbf{Z}$ or $\mathbf{Z}_{-}$and to rule out $\mathbf{Z}_{+}$is that for discrete-time filtrations, the interesting phenomena occur near time $-\infty$.

Among the invertible measure-preserving maps, Bernoulli shifts form a remarkable class. Similarly, the product-type filtrations (i.e., generated modulo the null sets by sequences of independent random variables) are considered as a well-understood class. The Brownian filtrations (generated modulo the null sets by Brownian motions) form a natural and widely studied class of continuous-time filtrations, although less simple.

Measure-preserving maps considered here will be taken on diffuse Lebesgue spaces. Various equivalent definitions of Lebesgue spaces are available. A simple definition of a Lebesgue space is a probability space which is isomorphic modulo the null sets to the union of some sub-interval of $[0,1]$, endowed with the Lebesgue $\sigma$-field and the Lebesgue measure, and a countable set of atoms. Most of the time, the Lebsegue space considered is non-atomic, so the sub-interval is $[0,1]$ itself. The class of Lebesgue spaces includes the completion of every Polish space. See [13] to find the main properties of Lebesgue spaces or [10] to get equivalent definitions. Working on Lebesgue spaces provides non-trivial measurability results, existence of generators... We recall in section 7 the definitions and the main properties of partitions, generators, entropy used in the present paper. 
Similarly, the filtrations considered here will be defined on a standard Borel probability space $(\Omega, \mathcal{F}, \mathbb{P})$, i.e. $(\Omega, \mathcal{F})$ is the Borel space associated to some Polish space, to ensure the existence of regular conditional probabilities. Given two sub- $\sigma$-fields $\mathcal{A}$ and $\mathcal{B}$, the inclusion $\mathcal{A} \subset \mathcal{B} \bmod \mathbb{P}$ means that for every $A \in \mathcal{A}$, there exists $B \in \mathcal{B}$ such that $\mathbb{P}(A \triangle B)=0$. We say that $\mathcal{A}$ and $\mathcal{B}$ are equal modulo the null sets (or modulo $\mathbb{P})$ when $\mathcal{A} \subset \mathcal{B} \bmod \mathbb{P}$ and $\mathcal{B} \subset \mathcal{A} \bmod \mathbb{P}$. We do not systematically complete the $\sigma$-fields to avoid troubles when working with conditional probabilities.

\subsection{Reminders on filtrations indexed by $\mathrm{Z}$ or $\mathrm{Z}_{-}$}

We now recall some classical but less known definitions and facts on filtrations. Given a filtration $\left(\mathcal{F}_{n}\right)_{n}$ indexed by $\mathbf{Z}$ or $\mathbf{Z}_{-}$, one says that $\left(\mathcal{F}_{n}\right)_{n}$ is product-type if $\left(\mathcal{F}_{n}\right)_{n}$ can be generated modulo $\mathbb{P}$ by some sequence $\left(I_{n}\right)_{n}$ of (independent) random variables.

One says that $\left(\mathcal{F}_{n}\right)_{n}$ has independent increments if there exists a sequence $\left(I_{n}\right)_{n}$ of random variables such that for every $n$ in $\mathbf{Z}$ or $\mathbf{Z}_{-}$,

$$
\mathcal{F}_{n}=\mathcal{F}_{n-1} \vee \sigma\left(I_{n}\right) \quad \bmod \mathbb{P} \text { and } I_{n} \text { is independent of } \mathcal{F}_{n-1} .
$$

Such a sequence $\left(I_{n}\right)_{n}$ is called a sequence of innovations and is necessarily a sequence of independent random variables.

One says that $\left(\mathcal{F}_{n}\right)_{n}$ is $\left(a_{n}\right)_{n}$-adic when it admits some sequence $\left(I_{n}\right)_{n}$ of innovations such that each $I_{n}$ is uniformly distributed on some finite set with size $a_{n}$. One says that $\left(\mathcal{F}_{n}\right)_{n}$ is poly-adic when $\left(\mathcal{F}_{n}\right)_{n}$ is $\left(a_{n}\right)_{n}$-adic for some sequence $\left(a_{n}\right)_{n}$ of positive integers, called adicity.

One says that $\left(\mathcal{F}_{n}\right)_{n}$ is Kolmogorovian if the tail $\sigma$-field $\mathcal{F}_{-\infty}:=\bigcap_{n} \mathcal{F}_{n}$ is trivial (i.e., contains only events with probability 0 or 1$)$.

By the definition and by Kolmogorov's zero-one law, any filtration indexed by $\mathbf{Z}$ or $\mathbf{Z}_{-}$must have independent increments and must be Kolmogorovian to be product-type. But Vershik showed in [27] that the converse is not true. A simple counter-example is given by Vershik's decimation process (example 2 in [27]). Actually Vershik worked with decreasing sequences of measurable partitions indexed by $\mathbf{Z}_{+}$and this frame was translated into filtrations indexed by $\mathbf{Z}_{-}$by M. Émery and W. Schachermayer [12].

\section{$1.3 \quad K$-automorphisms}

The Kolmogorov property for filtrations indexed by $\mathbf{Z}$ or $\mathbf{Z}_{-}$has an analogue for dynamical systems, although the definition is less simple in this frame: one says that an automorphism $T$ of a probability space $(Z, \mathcal{Z}, \pi)$ is a $K$-automorphism (or that $T$ has completely positive entropy) if for every $A \in \mathcal{Z}$, one has $h\left(T,\left\{A, A^{c}\right\}\right)>0$ whenever $0<\pi(A)<1$. This condition is nothing but the triviality of the $\sigma$-field

$$
\Pi(T):=\left\{A \in \mathcal{Z}: h\left(T,\left\{A, A^{c}\right\}\right)=0\right\},
$$

called Pinsker's factor. Actually, the 'events' of Pinsker's factor can be seen as the 'asymptotic events'. Indeed, if $\gamma$ is a countable generator of $(Z, \mathcal{Z}, \pi, T)$, then

$$
\Pi(T)=\overline{\bigcap_{n \geq 0} \bigvee_{k \geq n} T^{-k} \gamma}=\bar{\bigcap} \bigcap_{n \geq 0} \bigvee_{k \geq n} T^{k} \gamma,
$$


where the upper bar indicates completion with regard to $\pi$. To make the analogy clearer, set $\gamma=\left\{A_{\lambda}, \lambda \in \Lambda\right\}$. For each $x \in Z$, call $f(x) \in \Lambda$ the only index $\lambda$ such that $x \in A_{\lambda}$. For every $k \in \mathbf{Z}$, the $\sigma$-field generated by $T^{-k} \gamma$ is the $\sigma$-field associated to $f \circ T^{k}$ viewed as a $\Lambda$-valued random variable on $(Z, \mathcal{Z}, \pi)$. Therefore, $\Pi(T)$ is the asymptotic $\sigma$-field generated by the sequence $\left(f \circ T^{k}\right)_{k \geq 0}$.

\subsection{Content of the paper}

We have just viewed the analogy between the 'Kolmogorovianity' of a filtration indexed by $\mathbf{Z}_{-}$and the $K$-property of an automorphism of a Lebesgue space.

The next section is devoted to a parallel presentation of analogous notions and results in the three following contexts: automorphisms of Lebesgue spaces, filtrations indexed by $\mathbf{Z}_{-}$and Brownian filtrations. We investigate two notions - complementability and maximality - involving factors or poly-adic immersed filtrations or Brownian immersed filtrations according to the context. The results presented are essentially due to Ornstein and Weiss [21, Ornstein [20], and Thouvenot 25] for automorphisms of Lebesgue spaces; They come from [18] for filtrations indexed by $\mathbf{Z}_{-}$. They are due to Brossard, Émery and Leuridan [4, 5, 6] for Brownian filtrations.

Section 3 provides proofs of results on maximality which are not easy yo find in the literature. With some restrictions on the nature of the complement, complementability implies maximality. Section 4 is devoted to the proof of this implication. The converse was already known to be false for factors of automorphisms of Lebesgue spaces and for poly-adic immersed filtrations. In section 5, we provide a counter-example in the context of Brownian filtrations. The construction relies on a counter-example for polyadic immersed filtrations which is inspired by non-published notes of Tsirelson [26].

In spite of the similitude of the notions regardless the context, some differences exist. In section 6, we provide a non-complementable filtration (associated to a stationary process) yielding a complementable factor. This example is inspired by Vershik's decimation process (example 2 in [27]).

In section 7 , we recall the definitions and the main properties of partitions, generators, entropy used in the present paper.

\section{Parallel notions and results}

\subsection{Factors and Immersed filtrations}

Given an invertible measure preserving map $T$ of a Lebesgue space $(Z, \mathcal{Z}, \pi)$, we call factor of $T$, or more rigorously a factor of the dynamical system $(Z, \mathcal{Z}, \pi, T)$, any sub$\sigma$-field $\mathcal{B}$ of $\mathcal{Z}$ such that $T^{-1} \mathcal{B}=\mathcal{B}=T \mathcal{B} \bmod \pi$. Actually, the factor is the dynamical system $\left(Z, \mathcal{B},\left.\pi\right|_{\mathcal{B}}, T\right)$, which will be abbreviated in $(T, \mathcal{B})$ in the present paper. This definition of a factor is equivalent to the usual one. 1

\footnotetext{
${ }^{1}$ Actually, Rokhlin's theory ensures that if $\mathcal{B}$ is a factor of a Lebesgue space $(Z, \mathcal{Z}, \pi, T)$, then there exists a map $f$ from $Z$ to some Polish space $E$ such that $\mathcal{B}$ is generated up to the negligible events by the map $\Phi: x \mapsto\left(f\left(T^{k}(x)\right)\right)_{k \in \mathbf{Z}}$ from $Z$ to the product space $E^{\mathbf{Z}}$. Call $\nu=\Phi(\pi)=\pi \circ \Phi^{-1}$ the image measure of $\mu$ by $\Phi$. Then the completion $\left(E^{\mathbf{Z}}, \mathcal{B}\left(E^{\mathbf{Z}}\right), \nu\right)$ is a Lebesgue space, the shift operator $S:\left(y_{k}\right)_{k \in \mathbf{Z}} \mapsto\left(y_{k+1}\right)_{k \in \mathbf{Z}}$ is an automorphism of $E^{\mathbf{Z}}$, and $S \circ \Phi=\Phi \circ T$.

Conversely, if $(Y, \mathcal{Y}, \nu, S)$ is a dynamical system and $\Phi$ a measurable map from $Z$ to $Y$ such that $\Phi(\pi)=\nu$ and $S \circ \Phi=\Phi \circ T$, then the $\sigma$-field $\Phi^{-1}(\mathcal{Y})$ is a factor of $(Z, \mathcal{Z}, \pi, T)$.
} 
Given two filtrations $\left(\mathcal{U}_{t}\right)_{t \in \mathbb{T}}$ and $\left(\mathcal{Z}_{t}\right)_{t \in \mathbb{T}}$ on some probability space $(\Omega, \mathcal{A}, \mathbb{P})$, indexed by a common subset $\mathbb{T}$ of $\mathbb{R}$, one says that $\left(\mathcal{U}_{t}\right)_{t \in \mathbb{T}}$ is immersed in $\left(\mathcal{Z}_{t}\right)_{t \in \mathbb{T}}$ if every martingale in $\left(\mathcal{U}_{t}\right)_{t \in \mathbb{T}}$ is still a martingale in $\left(\mathcal{Z}_{t}\right)_{t \in \mathbb{T}}$. The notion of immersion is stronger than the inclusion. Actually, $\left(\mathcal{U}_{t}\right)_{t \in \mathbb{T}}$ is immersed in $\left(\mathcal{Z}_{t}\right)_{t \in \mathbb{T}}$ if and only if the two conditions below hold:

1. for every $t \in \mathbb{T}, \mathcal{U}_{t} \subset \mathcal{Z}_{t}$.

2. for every $s<t$ in $\mathbb{T}, \mathcal{U}_{t}$ and $\mathcal{Z}_{s}$ are independent conditionally on $\mathcal{U}_{s}$.

The additional condition means that the largest filtration does not give information in advance on the smallest one. We also make the useful following observation.

Lemma 1. Assume that $\left(\mathcal{U}_{t}\right)_{t \in \mathbb{T}}$ is immersed in $\left(\mathcal{Z}_{t}\right)_{t \in \mathbb{T}}$. Then $\left(\mathcal{U}_{t}\right)_{t \in \mathbb{T}}$ is completely determined (up to null sets) by its final $\sigma$-field

$$
\mathcal{U}_{\infty}:=\bigvee_{t \in \mathbb{T}} \mathcal{U}_{t}
$$

More precisely, $\mathcal{U}_{t}=\mathcal{U}_{\infty} \cap \mathcal{Z}_{t} \bmod \mathbb{P}$ for every $t \in \mathbb{T}$. In particular, if $\mathcal{U}_{\infty}=\mathcal{Z}_{\infty}$ $\bmod \mathbb{P}$, then $\mathcal{U}_{t}=\mathcal{Z}_{t} \bmod \mathbb{P}$ for every $t \in \mathbb{T}$. 2

When one works with Brownian filtrations, i.e. with filtrations generated by Brownian motions, then the immersion has many equivalent translations. The next statements are very classical (close statements are proved in [1) and they rely on the stochastic calculus and the predictable representation property of Brownian filtrations.

Proposition 2. Let $\left(B_{t}\right)_{t \geq 0}$ be a finite-dimensional Brownian motion adapted to some filtration $\left(\mathcal{Z}_{t}\right)_{t \geq 0}$, and $\left(\mathcal{B}_{t}\right)_{t \geq 0}$ its natural filtration. The following statements are equivalent.

1. $\left(B_{t}\right)_{t \geq 0}$ is a martingale in $\left(\mathcal{Z}_{t}\right)_{t \geq 0}$.

2. $\left(\mathcal{B}_{t}\right)_{t \geq 0}$ is immersed in $\left(\mathcal{Z}_{t}\right)_{t \geq 0}$.

3. For every $t \geq 0$, the process $B_{t+}-B_{t}$ is independent of $\mathcal{Z}_{t}$.

4. $\left(B_{t}\right)_{t \geq 0}$ is a Markov process in $\left(\mathcal{Z}_{t}\right)_{t \geq 0}$.

Definition 3. When these statements hold, we say that $\left(B_{t}\right)_{t \geq 0}$ is a Brownian motion in the filtration $\left(\mathcal{Z}_{t}\right)_{t \geq 0}$.

Note the analogy between the following two results.

Theorem 4 (Ornstein [19]). Every factor of a Bernoulli shift is equivalent to a Bernoulli shift.

Theorem 5 (Vershik [27]). If $\left(\mathcal{Z}_{n}\right)_{n \leq 0}$ is a product-type filtration such that the final sigma-field $\mathcal{Z}_{0}$ is essentially separable, then every poly-adic filtration immersed in $\left(\mathcal{Z}_{n}\right)_{n \leq 0}$ is product-type.

\footnotetext{
${ }^{2}$ The inclusion $\mathcal{U}_{t}=\mathcal{U}_{\infty} \cap \mathcal{Z}_{t}$ is immediate. To prove the converse, take $A \in \mathcal{U}_{\infty} \cap \mathcal{Z}_{t}$. Since $\mathcal{U}_{\infty}$ and $\mathcal{Z}_{t}$ are independent conditionally on $\mathcal{U}_{t}$, we get $\mathbb{P}\left[A \mid \mathcal{U}_{t}\right]=\mathbb{P}\left[A \mid \mathcal{Z}_{t}\right]=\mathbf{1}_{A}$ a.s., so $A \in \mathcal{U}_{t} \bmod \mathbb{P}$.
} 
One-dimensional Brownian filtrations can be viewed as continuous time versions of dyadic product-type filtrations. In this analogy, the predictable representation property of the continuous-time filtration corresponds to the dyadicity of the discrete-time filtration. Yet, the situation is much more involved when one works with Brownian filtrations, and the following question remains open.

If a filtration $\left(\mathcal{F}_{t}\right)_{t \geq 0}$ is immersed in some (possibly infinite-dimensional) Brownian filtration and has the predictable representation property with regard to some onedimensional Brownian motion $\beta$ (i.e., each martingale in $\left(\mathcal{F}_{t}\right)_{t \geq 0}$ can be obtained as a stochastic integral with regard to $\beta$ ), then is $\left(\mathcal{F}_{t}\right)_{t \geq 0}$ necessarily a Brownian filtration?

A partial answer was given by Émery (it follows from corollary 1 in [11]).

Theorem 6 (Émery [11]). Let $d \in \mathbf{N} \cup\{+\infty\}$. Assume that the filtration $\left(\mathcal{F}_{t}\right)_{t \geq 0}$ is $d$ Brownian after 0, i.e., there exists a d-dimensional Brownian motion $\left(B_{t}\right)_{t \geq 0}$ in $\left(\mathcal{F}_{t}\right)_{t \geq 0}$ such that for every $t \geq \varepsilon>0, \mathcal{F}_{t}$ is generated by $\mathcal{F}_{\varepsilon}$ and the increments $\left(B_{s}-B_{\varepsilon}\right)_{\varepsilon \leq s \leq t}$. If $\left(\mathcal{F}_{t}\right)_{t \geq 0}$ is immersed in some (possibly infinite-dimensional) Brownian filtration, then $\left(\mathcal{F}_{t}\right)_{t \geq 0}$ is a d-dimensional Brownian filtration.

In the statement, the role of the stronger hypothesis that $\left(\mathcal{F}_{t}\right)_{t>0}$ is Brownian after 0 is to guarantee that the difficulties arise only at time $0+$, so the situation gets closer to filtrations indexed by $\mathbf{Z}$ or $\mathbf{Z}_{-}$, for which the difficulties arise only at time $-\infty$.

\subsection{Complementability}

By complementability, we will mean the existence of some independent complement, although we will have to specify the nature of the complement.

The following definition is abridged from [21].

Definition 7. Let $(Z, \mathcal{Z}, \pi, T)$ be a Lebesgue dynamical system and $\mathcal{B}$ be a factor of $T$. One says that $\mathcal{B}$ is complementable if $\mathcal{B}$ possesses an independent complement in $(Z, \mathcal{Z}, \pi, T)$, i.e. a factor $\mathcal{C}$ of $T$ which is independent of $\mathcal{B}$ (with regard to $\pi$ ) such that $\mathcal{B} \vee \mathcal{C}=\mathcal{Z} \bmod \pi$

If $(Z, \mathcal{Z}, \pi, T)$ is the direct product of two dynamical systems $\left(Z_{1}, \mathcal{Z}_{1}, \pi_{1}, T_{1}\right)$ and $\left(Z_{2}, \mathcal{Z}_{2}, \pi_{2}, T_{2}\right)$, then $\mathcal{Z}_{1} \otimes\left\{\emptyset, Z_{2}\right\}$ and $\left\{\emptyset, Z_{1}\right\} \otimes \mathcal{Z}_{2}$ are factors of $(Z, \mathcal{Z}, \pi, T)$ and each of them is a complement of the other one. Now, let us look at a counterexample.

Example 8. Let $T$ be the Bernoulli shift on $Z=\{-1,1\}^{\mathbf{Z}}$ endowed with product $\sigma$-field $\mathcal{Z}$ and the uniform law. The map $\Phi: Z \rightarrow Z$ defined by $\Phi\left(\left(x_{n}\right)_{n \in \mathbf{Z}}\right)=\left(x_{n-1} x_{n}\right)_{n \in \mathbf{Z}}$ commutes with $T$, so $\Phi^{-1}(\mathcal{Z})$ is a factor of $T$. Call $p_{0}: Z \rightarrow\{-1,1\}$ the canonical projection defined by $p_{0}\left(\left(x_{n}\right)_{n \in \mathbf{Z}}\right)=x_{0}$. Then the $\sigma$-field $p_{0}^{-1}(\mathcal{Z})$ is an independent complement of $\Phi^{-1}(\mathcal{Z})$, but this complement is not a factor. Actually, we will come back to this example after definition 15 to show as an application of theorem 23 that no factor can be an independent complement of $\Phi^{-1}(\mathcal{Z})$.

We now define the notion of complementability in the world of filtrations.

Definition 9. Consider two filtrations $\left(\mathcal{U}_{t}\right)_{t \in \mathbb{T}}$ and $\left(\mathcal{Z}_{t}\right)_{t \in \mathbb{T}}$ on some probability space $(\Omega, \mathcal{A}, \mathbb{P})$, indexed by a common subset $\mathbb{T}$ of $\mathbb{R}$. One says that $\left(\mathcal{U}_{t}\right)_{t \in \mathbb{T}}$ is complementable in $\left(\mathcal{Z}_{t}\right)_{t \in \mathbb{T}}$ if there exists a filtration $\left(\mathcal{V}_{t}\right)_{t \in \mathbb{T}}$ such that for every $t \in \mathbb{T}, \mathcal{U}_{t}$ and $\mathcal{V}_{t}$ are independent and $\mathcal{U}_{t} \vee \mathcal{V}_{t}=\mathcal{Z}_{t} \bmod \mathbb{P}$. 
Since independent enlargements of a filtration always produce filtrations in which the initial filtration is immersed, $\left(\mathcal{U}_{t}\right)_{t \in \mathbb{T}}$ needs to be immersed in $\left(\mathcal{Z}_{t}\right)_{t \in \mathbb{T}}$ to possess an independent complement.

We will use many times the next result, abridged from [18].

Proposition 10. Keep the notations of the last definition. Let $U$ be a random variable valued in some measurable space $(E, \mathcal{E})$, such that $\sigma(U)=\bigvee_{t \in \mathbb{T}} \mathcal{U}_{t}$, and $\left(\mathbb{P}_{u}\right)_{u \in E}$ a regular version of the conditional probability $\mathbb{P}$ given $U$. Assume that $\left(\mathcal{U}_{t}\right)_{t \in \mathbb{T}}$ is complementable in $\left(\mathcal{Z}_{t}\right)_{t \in \mathbb{T}}$ by a filtration $\left(\mathcal{V}_{t}\right)_{t \in \mathbb{T}}$. Then for $U(\mathbb{P})$-almost every $u \in E$, the filtered probability space $\left(\Omega, \mathcal{A}, \mathbb{P}_{u},\left(\mathcal{Z}_{t}\right)_{t \leq \mathbb{T}}\right)$ is isomorphic to the filtered probability space $\left(\Omega, \mathcal{A}, \mathbb{P},\left(\mathcal{V}_{t}\right)_{t \leq \mathbb{T}}\right)$.

Let us give applications of the last result, that will be used in the present paper.

Corollary 11. (Particular cases)

- If a filtration $\left(\mathcal{U}_{n}\right)_{n \leq 0}$ is complementable in $\left(\mathcal{Z}_{n}\right)_{n \leq 0}$ by some product-type filtration, then for $U(\mathbb{P})$-almost every $u \in E,\left(\mathcal{Z}_{n}\right)_{n \leq 0}$ is product-type under $\mathbb{P}_{u}$.

- If a filtration $\left(\mathcal{U}_{t}\right)_{t \geq 0}$ is complementable in $\left(\mathcal{Z}_{t}\right)_{t \geq 0}$ by some Brownian filtration, then for $U(\mathbb{P})$-almost every $u \in E,\left(\mathcal{Z}_{t}\right)_{t \geq 0}$ is a Brownian filtration under $\mathbb{P}_{u}$.

Determining whether a 1-dimensional Brownian filtration immersed in a 2-dimensional Brownian filtration is complementable or not is often difficult. Except trivial cases, the only known cases are related to skew-product decomposition of the planar Brownian motion, see [6].

\subsection{Maximality}

The definition of the maximality requires a tool to measure the quantity of information. When one works with factors of an automorphism of a Lebesgue space, the quantity of information is the entropy. When one works with poly-adic filtrations, the quantity of information is the sequence of positive integers giving the adicity. When one works with Brownian filtrations, the quantity of information is the dimension of any generating Brownian motion. The classical statements below show hove these quantities vary when one considers a factor, a poly-adic immersed filtration, or a Brownian immersed filtration.

Remark 12. (Quantity of information in subsystems)

1. If $\mathcal{B}$ is a factor of $(Z, \mathcal{Z}, \pi, T)$, then $h(T, \mathcal{B}) \leq h(T)$.

2. If a $\left(b_{n}\right)_{n \leq 0}$-adic filtration is immersed in an $\left(r_{n}\right)_{n \leq 0}$-adic filtration, then $b_{n}$ divides $r_{n}$ for every $n$.

3. If a m-dimensional Brownian filtration is immersed in a n-dimensional Brownian filtration, then $m \leq n$.

The first statement is very classical. The second one is proved in [18]. The last one is classical and shows that the dimension of a Brownian filtration makes sense; a proof is given in the footnote. 3

\footnotetext{
${ }^{3}$ Let $Z$ be a $n$-dimensional Brownian motion and $B$ be a $m$-dimensional Brownian motion in $\mathcal{F}^{Z}$. Then one can find an $\mathcal{F}^{Z}$-predictable process $M$ taking values in the set of all $p \times n$ real matrices whose lines form an orthonormal family, such that $B=\int_{0}^{*} M_{s} \mathrm{~d} Z_{s}$. In particular, the $m$ lines of each matrix $M_{s}$ are independent and lie in a $n$-dimensional vector space, so $m \leq n$.
} 
Let us give precise definitions, respectively abridged from [20], [18] and [4] or [5].

Definition 13. Let $(Z, \mathcal{Z}, \pi, T)$ be a Lebesgue dynamical system and $\mathcal{B}$ be a factor of $T$. One says that $\mathcal{B}$ is maximal if $(T, \mathcal{B})$ has a finite entropy and if for any factor $\mathcal{A}$, the conditions $\mathcal{A} \supset \mathcal{B}$ and $h(T, \mathcal{A})=h(T, \mathcal{B})$ entail $\mathcal{A}=\mathcal{B}$ modulo null sets.

Definition 14. Let $\left(\mathcal{B}_{n}\right)_{n \leq 0}$ be a $\left(b_{n}\right)_{n \leq 0}$-adic filtration immersed in some filtration $\left(\mathcal{Z}_{n}\right)_{n \leq 0}$. One says that $\left(\mathcal{B}_{n}\right)_{n \leq 0}$ is maximal in $\left(\mathcal{Z}_{n}\right)_{n \leq 0}$ if every $\left(b_{n}\right)_{n \leq 0}$-adic filtration immersed in $\left(\mathcal{Z}_{n}\right)_{n \leq 0}$ and containing $\left(\mathcal{B}_{n}\right)_{n \leq 0}$ is equal to $\left(\mathcal{B}_{n}\right)_{n \leq 0}$ modulo null events.

Definition 15. Let $\left(\mathcal{B}_{t}\right)_{t \geq 0}$ be a d-dimensional Brownian filtration immersed in some filtration $\left(\mathcal{Z}_{t}\right)_{t>0}$. One says that $\left(\mathcal{B}_{t}\right)_{t>0}$ is maximal in $\left(\mathcal{Z}_{t}\right)_{t>0}$ if every d-dimensional Brownian filtration immersed in $\left(\mathcal{Z}_{t}\right)_{t \geq 0}$ and containing $\left(\mathcal{B}_{t}\right)_{t \leq 0}$ is equal to $\left(\mathcal{B}_{t}\right)_{t \geq 0}$ modulo null events.

Let us come back to example 8 in which $T$ be the Bernoulli shift on $Z=\{-1,1\}^{\mathbf{Z}}$ endowed with product $\sigma$-field $\mathcal{Z}$ and the uniform law. Since the map $\Phi: Z \rightarrow Z$ defined by $\Phi\left(\left(x_{n}\right)_{n \in \mathbf{Z}}\right)=\left(x_{n-1} x_{n}\right)_{n \in \mathbf{Z}}$ commutes with $T$ and preserves the uniform law on $Z$, the factor $\left(T, \Phi^{-1}(\mathcal{Z})\right)$ is a Bernoulli $(1 / 2,1 / 2)$ shift like $T$ itself. The factor $\Phi^{-1}(\mathcal{Z})$ is strictly contained in $\mathcal{Z}$ but has the same (finite) entropy as $T$, so it is not maximal. But every factor of $T$ is a $K$-automorphism since $T$ is. Hence, theorem 23 will show that the factor $\Phi^{-1}(\mathcal{Z})$ is not complementable.

This example above can be abridged in the context of filtrations indexed by the relative integers : consider a sequence $\left(\xi_{n}\right)_{n \in \mathbf{Z}}$ of independent uniform random variables taking values in $\{-1,1\}$. Then the sequence $\left(\eta_{n}\right)_{n \in \mathbf{Z}}:=\Phi\left(\left(\xi_{n}\right)_{n \in \mathbf{Z}}\right)$ has the same law as $\left(\xi_{n}\right)_{n \in \mathbf{Z}}$. One checks that the inclusions $\mathcal{F}_{n}^{\eta} \subset \mathcal{F}_{n}^{\xi}$ are strict modulo $\mathbb{P}$, although the tail $\sigma$-field $\mathcal{F}_{-\infty}^{\xi}$ are trivial and although $\left(\eta_{n}\right)_{n \in \mathbf{Z}}$ is an innovation sequence for $\left(\mathcal{F}_{n}^{\xi}\right)_{n \in \mathbf{Z}}$. Actually, one bit of information is lost when one transforms $\left(\xi_{n}\right)_{n \in \mathbf{Z}}$ into $\left(\eta_{n}\right)_{n \in \mathbf{Z}}$ : for each $n_{0} \in \mathbf{Z}$, the value $\xi_{n_{0}}$ is independent of $\left(\eta_{n}\right)_{n \in \mathbf{Z}}$, and the knowledge of $\xi_{n_{0}}$ and $\left(\eta_{n}\right)_{n \in \mathbf{Z}}$ is sufficient to recover $\left(\xi_{n}\right)_{n \in \mathbf{Z}}$. The paradox is that this loss of information is asymptotic at time $-\infty$ but invisible when one looks at $\mathcal{F}_{-\infty}^{\xi}$ and $\mathcal{F}_{-\infty}^{\eta}$.

The situation can be much more complex when one works with Brownian filtrations. For example, consider a linear Brownian motion $W$. Since $W$ spends a null-time at 0 , the stochastic integral

$$
W^{\prime}=\int_{0}^{\cdot} \operatorname{sgn}\left(W_{s}\right) \mathrm{d} W_{s}=|W|-L
$$

(where $L$ denotes the local time of $W$ at 0 ) is still a linear Brownian motion. The natural filtration $\mathcal{F}^{W^{\prime}}$ is immersed and strictly included in $\mathcal{F}^{W}$, therefore it is not maximal in $\mathcal{F}^{W}$. Actually, $W^{\prime}$ generates the same filtration as $|W|$ up to null events, so the Lévy transformation - which transforms the sample paths of $W$ into the sample paths of $W^{\prime}$ - forgets the signs of all excursions of $W$, which are independent of $|W|$. Here, the loss of information occurs at each beginning of excursion of $W$, and not at time $0+$.

\subsection{Necessary or sufficient conditions for maximality}

Given a finite-entropy factor, a poly-adic immersed filtration or a Brownian immersed filtration, one wishes to enlarge it to get a maximal one having the same entropy, adicity or dimension. This leads to the following constructions, abridged from [20, 18, 5]. In the next three propositions, the bars above the $\sigma$-fields indicate completions with regard to $\pi$ or $\mathbb{P}$. 
Definition 16. Let $(Z, \mathcal{Z}, \pi)$ be a probability space, $T$ be an invertible measure-preserving map on $(Z, \mathcal{Z}, \pi)$ and $\mathcal{B}$ be a factor with finite entropy. The conditional Pinsker factor associated to $\mathcal{B}$ is defined by

$$
\mathcal{B}^{\prime}:=\left\{A \in \mathcal{Z}: h\left(T,\left\{A, A^{c}\right\} \mid \mathcal{B}\right)=0\right\} .
$$

where

$$
h\left(T,\left\{A, A^{c}\right\} \mid \mathcal{B}\right)=\lim _{n \rightarrow+\infty} \frac{1}{n} H\left(\bigvee_{k=0}^{n-1}\left\{T^{-k} A, T^{-k} A^{c}\right\} \mid \mathcal{B}\right) .
$$

Proposition 17. The collection $\mathcal{B}^{\prime}$ thus defined is the largest factor containing $\mathcal{B}$ and having the same entropy as $\mathcal{B}$. In particular, $\mathcal{B}^{\prime}$ is maximal.

Proposition 18. Furthermore, assume $(Z, \mathcal{Z}, \pi, T)$ is a Lebesgue dynamical space, that $T$ is aperiodic 4 and has finite entropy. Then for every generator $\gamma$ of $T$,

$$
\mathcal{B}^{\prime}=\overline{\bigcap_{n \geq 0}\left(\mathcal{B} \vee \bigvee_{k \geq n} T^{-k} \gamma\right)}
$$

Proposition 19. Let $\left(\mathcal{B}_{n}\right)_{n \leq 0}$ be a $\left(b_{n}\right)_{n \leq 0}$-adic filtration immersed in some filtration $\left(\mathcal{Z}_{n}\right)_{n \leq 0}$. Then $\left(\mathcal{B}_{n}\right)_{n \leq 0}$ is immersed in the filtration $\left(\mathcal{B}_{n}^{\prime}\right)_{n \leq 0}$ defined by

$$
\mathcal{B}_{n}^{\prime}:=\overline{\bigcap_{s \leq 0}\left(\mathcal{B}_{n} \vee \mathcal{Z}_{s}\right)} .
$$

Moreover $\left(\mathcal{B}_{n}^{\prime}\right)_{n \leq 0}$ is the largest $\left(b_{n}\right)_{n \leq 0}$-adic filtration containing $\left(\mathcal{B}_{n}\right)_{n \leq 0}$ and immersed in $\left(\mathcal{Z}_{n}\right)_{n \leq 0}$. In particular, $\left(\mathcal{B}_{n}^{\prime}\right)_{n \leq 0}$ is maximal in $\left(\mathcal{Z}_{n}\right)_{n \leq 0}$.

Proposition 20. Let $\left(\mathcal{B}_{t}\right)_{t \geq 0}$ be a d-dimensional Brownian filtration immersed in some Brownian filtration $\left(\mathcal{Z}_{t}\right)_{t \geq 0}$. Then $\left(\mathcal{B}_{t}\right)_{t \geq 0}$ is immersed in the filtration $\left(\mathcal{B}_{t}^{\prime}\right)_{t \geq 0}$ defined by

$$
\mathcal{B}_{t}^{\prime}:=\overline{\bigcap_{s>0}\left(\mathcal{B}_{t} \vee \mathcal{Z}_{s}\right)}
$$

Moreover $\left(\mathcal{B}_{t}^{\prime}\right)_{t \geq 0}$ is a d-dimensional Brownian filtration immersed in $\left(\mathcal{Z}_{t}\right)_{t \geq 0}$.

Be careful: when $\mathcal{F}$ is a sub- $\sigma$-field and $\left(\mathcal{G}_{n}\right)_{n \geq 0}$ is a non-increasing sequence of sub- $\sigma$-fields of a probability space $(\Omega, \mathcal{T}, \mathbb{P})$, the trivial inclusion

$$
\mathcal{F} \vee\left(\bigcap_{n \geq 0} \mathcal{G}_{n}\right) \subset \bigcap_{n \geq 0}\left(\mathcal{F} \vee \mathcal{G}_{n}\right)
$$

may be strict modulo $\mathbb{P}$. Equality modulo $\mathbb{P}$ holds when $\mathcal{F}$ and $\mathcal{G}_{0}$ are independent (see corollary (39). Von Weizsäcker provides involved characterizations in 28. Therefore, the $\sigma$-fields $\mathcal{B}^{\prime}, \mathcal{B}_{n}^{\prime}$ and $\mathcal{B}_{t}^{\prime}$ considered in propositions [18, 19, and 20, can be strictly larger than the $\sigma$-fields $\overline{\mathcal{B} \vee \Pi(T)}, \overline{\mathcal{B}_{n} \vee \mathcal{Z}_{-\infty}}$ and $\overline{\mathcal{B}_{t} \vee \mathcal{Z}_{0+}}=\overline{\mathcal{B}_{t}}$ respectively.

Note the analogy between the formulas in propositions [18, 19, and 20. In these three contexts, we must have $\mathcal{B}^{\prime}=\mathcal{B}$ up to null sets for $\mathcal{B}$ to be maximal. Moreover, applying the same procedure to $\mathcal{B}^{\prime}$ leads to $\mathcal{B}^{\prime \prime}=\mathcal{B}^{\prime}$. Hence the condition $\mathcal{B}^{\prime}=\mathcal{B}$ up to null sets is also sufficient for $\mathcal{B}$ to be maximal in the first two cases (factors of finite-entropy aperiodic Lebesgue automorphisms and poly-adic filtrations). But once again, the situation is more complex when one works with Brownian filtrations, since the filtration $\mathcal{B}^{\prime}$ may be non-maximal. Here is a counter-example (the proof will be given in section 3 ).

\footnotetext{
${ }^{4}$ Aperiodicity of $T$ means that $\pi\left\{z \in Z: \exists n \geq 1, T^{n}(z)=z\right\}=0$. We make this assumption to ensure the existence of generator.
} 
Example 21. Let $X$ be a linear Brownian motion in some filtration $\mathcal{Z}$. Set

$$
B=\int_{0}^{\cdot} \operatorname{sgn}\left(X_{s}\right) \mathrm{d} X_{s}
$$

and call $\mathcal{X}$ and $\mathcal{B}$ the natural filtrations of $X$ and $B$. If $\mathcal{X}$ is maximal in $\mathcal{Z}$, then the filtration $\mathcal{B}^{\prime}$ defined by proposition 20 coincides with $\mathcal{B}$ up to null events. Therefore, the filtration $\mathcal{B}^{\prime}$ (included in $\mathcal{X}$ ) cannot be maximal in $\mathcal{Z}$.

Actually, the maximality of Brownian filtrations is not an asymptotic property at $0+$, unlike the almost sure equality $\mathcal{B}^{\prime}=\mathcal{B}$. To try to produce a maximal Brownian filtration containing a given Brownian filtration, one should perform the infinitesimal enlargement above at every time, but we do not see how to do that.

Yet, proposition 27 in the next subsection shows that that equality $\mathcal{B}=\mathcal{B}^{\prime}$ ensures the maximality of $\mathcal{B}$ under the (strong) additional hypothesis that $\mathcal{B}$ is complementable after 0 .

The next sufficient condition for the maximality of a poly-adic immersed filtration comes from [18].

Proposition 22. Let $\left(\mathcal{B}_{n}\right)_{n \leq 0}$ be a $\left(b_{n}\right)_{n \leq 0}$-adic filtration immersed in $\left(\mathcal{Z}_{n}\right)_{n \leq 0}$. Let $U$ be a random variable valued in some measurable space $(E, \mathcal{E})$, such that $\sigma(U)=\mathcal{B}_{0}$ and $\left(\mathbb{P}_{u}\right)_{u \in E}$ a regular version of the conditional probability $\mathbb{P}$ given $U$. If for $U(\mathbb{P})$-almost every $u \in E$, the filtered probability space $\left(\Omega, \mathcal{A}, \mathbb{P}_{u},\left(\mathcal{Z}_{n}\right)_{n \leq 0}\right)$ is Kolmogorovian, then the filtration $\left(\mathcal{B}_{n}\right)_{n \leq 0}$ is maximal in $\left(\mathcal{Z}_{n}\right)_{n \leq 0}$.

The assumption that $\left(\mathcal{Z}_{n}\right)_{n \leq 0}$ is Kolmogorovian under almost every conditional probability $\mathbb{P}_{u}$ echoes to the alternative terminology of conditional $K$-automorphisms used by Thouvenot in [25].

\subsection{Complementability and maximality}

In the three contexts (factors of an automorphism of a Lebsgue space, poly-adic filtrations immersed in a filtration indexed by $\mathbf{Z}_{-}$, Brownian filtrations immersed in a Brownian filtration), we get very similar results.

The first one is stated by Ornstein in [20] as a direct consequence of a lemma stated in [21].

Proposition 23. Assume that $T$ with finite entropy. Let $\mathcal{B}$ be a factor of $T$. If $\mathcal{B}$ is complementable by some $K$-automorphism, then $\mathcal{B}$ is maximal.

The second one comes from [18].

Proposition 24. Let $\left(\mathcal{B}_{n}\right)_{n \leq 0}$ be a $\left(b_{n}\right)_{n \leq 0}$-adic filtration immersed in $\left(\mathcal{Z}_{n}\right)_{n \leq 0}$. If $\left(\mathcal{B}_{n}\right)_{n \leq 0}$ can be complemented by some Kolmogorovian filtration, then $\left(\mathcal{B}_{n}\right)_{n \leq 0}$ is maximal in $\left(\mathcal{Z}_{n}\right)_{n \leq 0}$.

A particular case of the third one (in which the dimension of the Brownian filtrations are 1 and 2) can be found in 44 or [5].

Proposition 25. Let $\left(\mathcal{B}_{t}\right)_{t \geq 0}$ be a Brownian filtration immersed in a Brownian filtration $\left(\mathcal{Z}_{t}\right)_{t \geq 0}$ with larger dimension. If $\left(\mathcal{B}_{t}\right)_{t \geq 0}$ can be complemented by some Brownian filtration, then $\left(\mathcal{B}_{t}\right)_{t \geq 0}$ is maximal in $\left(\mathcal{Z}_{t}\right)_{t \geq 0}$. 
The proofs of these three statements are rather simple, and present some similarities, although they are different. In the next section, we provide two different proofs of proposition 23. The first one relies on Ornstein and Weiss' lemma (lemma 2 in [21]). The second one is a bit simpler but requires that $T$ has finite entropy, and relies on Berg's lemma (lemma 2.3 in [3]). We also provide a proof of proposition 25. Proposition 24] follows from proposition 22 and corollary 11

The converses of the three implications above are false, but providing counter-examples is difficult. Ornstein gived in 20] an example of maximal but non-complementable factor in [20], but the proof is difficult to read. Two counter-examples of a maximal but non-complementable poly-adic filtration are given in [18. In the present paper, we use a third example to construct a maximal but non-complementable Brownian filtration.

In the present paper, we will also use a small refinement of proposition 25, using the notion of complementability after 0 .

Definition 26. Let $\left(\mathcal{B}_{t}\right)_{t \geq 0}$ be a Brownian filtration immersed in a Brownian filtration $\left(\mathcal{Z}_{t}\right)_{t \geq 0}$ with larger dimension. One says that $\left(\mathcal{B}_{t}\right)_{t \geq 0}$ is complementable after 0 in $\left(\mathcal{Z}_{t}\right)_{t \geq 0}$ if there exists some Brownian filtration $\mathcal{C}$ immersed in $\mathcal{Z}$ and independent of $\mathcal{B}$ such that,

$$
\forall t \geq 0, \mathcal{Z}_{t}=\bigcap_{s>0}\left(\mathcal{B}_{t} \vee \mathcal{C}_{t} \vee \mathcal{Z}_{s}\right) \bmod \pi
$$

Proposition 27. Let $\left(\mathcal{B}_{t}\right)_{t>0}$ be a d-dimensional Brownian filtration immersed in a Brownian filtration $\left(\mathcal{Z}_{t}\right)_{t \geq 0}$ with larger dimension. If $\left(\mathcal{B}_{t}\right)_{t \leq 0}$ is complementable after 0 , then the filtration provided by proposition 20 is the largest d-dimensional Brownian filtration immersed in $\left(\mathcal{Z}_{t}\right)_{t \leq 0}$ and containing $\left(\mathcal{B}_{t}\right)_{t \leq 0}$. In particular, $\left(\mathcal{B}_{t}^{\prime}\right)_{t \leq 0}$ is maximal in $\left(\mathcal{Z}_{t}\right)_{t \leq 0}$.

\section{Conditions for maximality: proofs}

In this section, we provide proofs of the statements given in subsection 2.4, except proposition 19 which is proved in [18].

\subsection{Proof of proposition 17}

By definition, $\mathcal{B}^{\prime}$ is closed under taking complements. For every $A$ and $B$ in $\mathcal{Z}$, the partition $\left\{A \cup B,(A \cup B)^{c}\right\}$ is less fine that $\left\{A, A^{c}\right\} \vee\left\{B, B^{c}\right\}$, hence

$$
\begin{aligned}
h\left(T,\left\{A \cup B,(A \cup B)^{c}\right\} \mid \mathcal{B}\right) & \leq h\left(T,\left\{A, A^{c}\right\} \vee\left\{B, B^{c}\right\} \mid \mathcal{B}\right) \\
& \leq h\left(T,\left\{A, A^{c}\right\} \mid \mathcal{B}\right)+h\left(T,\left\{B, B^{c}\right\} \mid \mathcal{B}\right) .
\end{aligned}
$$

One deduce that $\mathcal{B}^{\prime}$ is closed under finite union.

But $h\left(T,\left\{A, A^{c}\right\} \mid \mathcal{B}\right)$ depends continuously on $A$ when $\mathcal{Z}$ is endowed with the pseudometric defined by $\delta(A, B)=\pi(A \triangle B)$ (see proposition [74), so $\mathcal{B}^{\prime}$ is a closed subset. Hence, $\mathcal{B}^{\prime}$ is a complete $\sigma$-field.

The equalities $h\left(T,\left\{A, A^{c}\right\} \mid \mathcal{B}\right)=h\left(T,\left\{T^{-1} A, T^{-1} A^{c}\right\} \mid \mathcal{B}\right)=h\left(T,\left\{T A, T A^{c}\right\} \mid \mathcal{B}\right)$ for every $A \in \mathcal{Z}$ show that $\mathcal{B}^{\prime}$ is a factor.

Moreover, $\mathcal{B} \subset \mathcal{B}^{\prime}$ since for every $B \in \mathcal{B}, h\left(T,\left\{B, B^{c}\right\} \mid \mathcal{B}\right) \leq H\left(\left\{B, B^{c}\right\} \mid \mathcal{B}\right)=0$. 
The sub-additivity of entropy shows that $h(T, \alpha \mid \mathcal{B})=0$ for every finite partition $\alpha \subset \mathcal{B}^{\prime}$. Hence $h\left(T, \mathcal{B}^{\prime}\right)-h(T, \mathcal{B})=h\left(\left(T, \mathcal{B}^{\prime}\right) \mid \mathcal{B}\right)=0$.

Last, let $\mathcal{A}$ be a factor containing $\mathcal{B}$ and having the same entropy as $\mathcal{B}$. Then for every $A \in \mathcal{A}$,

$$
h\left(T,\left\{A, A^{c}\right\} \mid \mathcal{B}\right) \leq h((T, \mathcal{A}) \mid \mathcal{B})=h(T, \mathcal{A})-h(T, \mathcal{B})=0,
$$

so $\mathcal{A} \subset \mathcal{B}^{\prime}$. The proof is complete.

\subsection{Proof of proposition 18}

The proofs given here are inspired by the proofs of the similar results involving (nonconditional) Pinsker factor given in 9.

For every countable measurable partition $\alpha$ of $(Z, \mathcal{Z}, \pi)$ and for every integers $p \leq q$, we introduce the notations

$$
\alpha_{p}^{q}:=\bigvee_{k=p}^{q} T^{-k} \alpha, \quad \alpha_{1}^{\infty}=\bigvee_{k \geq 1} T^{-k} \alpha, \quad \mathcal{B}^{\alpha}:=\bigcap_{n \geq 0}\left(\mathcal{B} \vee \bigvee_{k \geq n} T^{-k} \alpha\right)
$$

Let us recall that the inclusion

$$
\mathcal{B}^{\alpha} \supset \mathcal{B} \vee \bigcap_{n \geq 0}\left(\bigvee_{k \geq n} T^{-k} \alpha\right)
$$

can be strict modulo $\mathbb{P}$. We also note that the larger is $n$, the smaller is the partition

$$
T^{-n} \alpha_{1}^{\infty}=\bigvee_{k \geq n+1} T^{-k} \alpha
$$

so $\mathcal{B}^{\alpha}$ is also the intersection of the non-increasing sequence $\left(\mathcal{B} \vee T^{-n} \alpha_{1}^{\infty}\right)_{n \geq 0}$.

We begin with the following lemma.

Lemma 28. Let $\alpha$ and $\gamma$ be countable measurable partitions of $(Z, \mathcal{Z}, \pi)$, with finite entropy. Then $H\left(\alpha \mid \alpha_{1}^{\infty} \vee \mathcal{B}^{\gamma}\right)=H\left(\alpha \mid \alpha_{1}^{\infty} \vee \mathcal{B}\right)$.

Proof. The addition formula for conditional entropy yields for every $n \geq 1$,

$$
H\left(\alpha \vee \cdots \vee T^{n-1} \alpha \mid \alpha_{1}^{\infty} \vee \mathcal{B}\right)=\sum_{k=0}^{n-1} H\left(T^{k} \alpha \mid T^{k} \alpha_{1}^{\infty} \vee \mathcal{B}\right)=n H\left(\alpha \mid \alpha_{1}^{\infty} \vee \mathcal{B}\right)
$$

Replacing $\alpha$ with $\alpha \vee \gamma$ gives

$$
U_{n}:=H\left(\alpha_{-n+1}^{0} \vee \gamma_{-n+1}^{0} \mid \alpha_{1}^{\infty} \vee \mathcal{B} \vee \gamma_{1}^{\infty}\right)=n H\left(\alpha \vee \gamma \mid \alpha_{1}^{\infty} \vee \mathcal{B} \vee \gamma_{1}^{\infty}\right)
$$

Set

$$
V_{n}:=H\left(\alpha_{-n+1}^{0} \vee \gamma_{-n+1}^{0} \mid \alpha_{1}^{\infty} \vee \mathcal{B}\right) \text { and } W_{n}:=H\left(\alpha_{-n+1}^{0} \vee \gamma_{-n+1}^{0} \mid \mathcal{B}\right)
$$

Since $U_{n} \leq V_{n} \leq W_{n}$ and

$$
\lim _{n} W_{n} / n=h((T, \alpha \vee \gamma) \mid \mathcal{B})=H\left(\alpha \vee \gamma \mid \alpha_{1}^{\infty} \vee \mathcal{B} \vee \gamma_{1}^{\infty}\right),
$$

we get $\lim _{n} U_{n} / n=\lim _{n} V_{n} / n$. But

$$
U_{n}=H\left(\alpha_{-n+1}^{0} \mid \alpha_{1}^{\infty} \vee \mathcal{B} \vee \gamma_{1}^{\infty}\right)+H\left(\alpha_{-n+1}^{0} \vee \gamma_{-n+1}^{0} \mid T^{n} \alpha_{1}^{\infty} \vee \mathcal{B} \vee \gamma_{1}^{\infty}\right),
$$




$$
V_{n}=H\left(\alpha_{-n+1}^{0} \mid \alpha_{1}^{\infty} \vee \mathcal{B}\right)+H\left(\alpha_{-n+1}^{0} \vee \gamma_{-n+1}^{0} \mid T^{n} \alpha_{1}^{\infty} \vee \mathcal{B}\right)
$$

In these two expressions, each term in $U_{n}$ is less or equal that the corresponding term in $V_{n}$. Since $H\left(\alpha_{-n+1}^{0} \mid \alpha_{1}^{\infty} \vee \mathcal{B}\right)=n H\left(\alpha \mid \alpha_{1}^{\infty} \vee \mathcal{B}\right)$ we derive that

$$
\lim _{n} n^{-1} H\left(\alpha_{-n+1}^{0} \mid \alpha_{1}^{\infty} \vee \mathcal{B} \vee \gamma_{1}^{\infty}\right)=H\left(\alpha \mid \alpha_{1}^{\infty} \vee \mathcal{B}\right)
$$

Furthermore, we note that

$$
\alpha_{1}^{\infty} \vee \mathcal{B}^{\gamma} \subset \bigcap_{n \geq 0}\left(\alpha_{1}^{\infty} \vee \mathcal{B} \vee T^{-n} \gamma_{1}^{\infty}\right)
$$

so proposition 61. Cesàro's lemma and addition formula for conditional entropy yield

$$
\begin{aligned}
H\left(\alpha \mid \alpha_{1}^{\infty} \vee \mathcal{B}^{\gamma}\right) & \geq \lim _{n} H\left(\alpha \mid \alpha_{1}^{\infty} \vee \mathcal{B} \vee T^{-n} \gamma_{1}^{\infty}\right) \\
& =\lim _{n} H\left(T^{n} \alpha \mid T^{n} \alpha_{1}^{\infty} \vee \mathcal{B} \vee \gamma_{1}^{\infty}\right) \\
& =\lim _{n} n^{-1} \sum_{k=0}^{n-1} H\left(T^{k} \alpha \mid T^{k} \alpha_{1}^{\infty} \vee \mathcal{B} \vee \gamma_{1}^{\infty}\right) \\
& =\lim _{n} n^{-1} H\left(\alpha_{-n+1}^{0} \mid \alpha_{1}^{\infty} \vee \mathcal{B} \vee \gamma_{1}^{\infty}\right) \\
& =H\left(\alpha \mid \alpha_{1}^{\infty} \vee \mathcal{B}\right) .
\end{aligned}
$$

But the reverse inequality follows from the inclusion $\alpha_{1}^{\infty} \vee \mathcal{B} \subset \alpha_{1}^{\infty} \vee \mathcal{B}^{\gamma}$. Hence the equality holds.

Lemma 28 yields one inclusion in the equality of proposition 18, thanks to the next corollary.

Corollary 29. For every countable measurable partition $\gamma$ of $(Z, \mathcal{Z}, \pi)$, with finite entropy, $\overline{\mathcal{B}^{\gamma}} \subset \mathcal{B}^{\prime}$.

Proof. Since $\mathcal{B}^{\prime}$ is complete, one only needs to check that $\mathcal{B}^{\gamma} \subset \mathcal{B}^{\prime}$. Let $A \in \mathcal{B}^{\gamma}$ and $\alpha=\left\{A, A^{c}\right\}$. Lemma 28 yields $h((T, \alpha) \mid \mathcal{B})=H\left(\alpha \mid \alpha_{1}^{\infty} \vee \mathcal{B}\right)=H\left(\alpha \mid \alpha_{1}^{\infty} \vee \mathcal{B}^{\gamma}\right)=0$, so $A \in \mathcal{B}^{\prime}$. The inclusion follows.

Lemma 28 will also help us to prove the next useful lemma.

Lemma 30. Let $\alpha$ and $\gamma$ be countable measurable partitions of $(Z, \mathcal{Z}, \pi)$, with finite entropy. Let $N \geq 0$ and $\eta$ be a finite partition less fine that $\gamma_{-N}^{N}=\bigvee_{k=-N}^{N} T^{-k} \gamma$. Then

$$
H\left(\eta \mid\left(\mathcal{B}^{\alpha}\right)^{\gamma}\right)=H\left(\eta \mid \mathcal{B}^{\gamma} \vee \mathcal{B}^{\alpha}\right)=H\left(\eta \mid \mathcal{B}^{\gamma}\right) .
$$

Proof. For every $n \geq 0,\left(\gamma_{-n}^{n}\right)_{1}^{\infty}=T^{n} \gamma_{1}^{\infty}$, so $H\left(\gamma_{-n}^{n} \mid T^{n} \gamma_{1}^{\infty} \vee \mathcal{B}^{\alpha}\right)=H\left(\gamma_{-n}^{n} \mid T^{n} \gamma_{1}^{\infty} \vee \mathcal{B}\right)$ by lemma 28, When $n \geq N, \gamma_{-n}^{n}=\eta \vee \gamma_{-n}^{n}$, so

$$
\begin{gathered}
H\left(\gamma_{-n}^{n} \mid T^{n} \gamma_{1}^{\infty} \vee \mathcal{B}^{\alpha}\right)=H\left(\eta \mid T^{n} \gamma_{1}^{\infty} \vee \mathcal{B}^{\alpha}\right)+H\left(\gamma_{-n}^{n} \mid T^{n} \gamma_{1}^{\infty} \vee \mathcal{B}^{\alpha} \vee \eta\right), \\
H\left(\gamma_{-n}^{n} \mid T^{n} \gamma_{1}^{\infty} \vee \mathcal{B}\right)=H\left(\eta \mid T^{n} \gamma_{1}^{\infty} \vee \mathcal{B}\right)+H\left(\gamma_{-n}^{n} \mid T^{n} \gamma_{1}^{\infty} \vee \mathcal{B} \vee \eta\right)
\end{gathered}
$$

Since $H\left(\beta \mid \mathcal{A} \vee \mathcal{B}^{\alpha}\right) \leq H(\beta \mid \mathcal{A} \vee \mathcal{B})$ for every countable measurable partition $\beta$ and every $\sigma$-field $\mathcal{A} \subset \mathcal{Z}$, we get $H\left(\eta \mid T^{n} \gamma_{1}^{\infty} \vee \mathcal{B}^{\alpha}\right)=H\left(\eta \mid T^{n} \gamma_{1}^{\infty} \vee \mathcal{B}\right)$. Letting $n$ go to infinity yields $H\left(\eta \mid\left(\mathcal{B}^{\alpha}\right)^{\gamma}\right)=H\left(\eta \mid \mathcal{B}^{\gamma}\right)$. Since $\mathcal{B}^{\gamma} \subset \mathcal{B}^{\gamma} \vee \mathcal{B}^{\alpha} \subset\left(\mathcal{B}^{\alpha}\right)^{\gamma}$, the result follows. 
Corollary 31. Assume that $T$ has finite entropy and admits a generator $\gamma$. Then for every countable measurable partition $\alpha, \mathcal{B}^{\alpha} \subset \mathcal{B}^{\gamma}=\left(\mathcal{B}^{\gamma}\right)^{\gamma} \bmod \pi$.

Proof. The collection of all $A \in \mathcal{Z}$ such that

$$
H\left(\left\{A, A^{c}\right\} \mid \mathcal{B}^{\gamma}\right)=H\left(\left\{A, A^{c}\right\} \mid \mathcal{B}^{\gamma} \vee \mathcal{B}^{\alpha}\right)=H\left(\left\{A, A^{c}\right\} \mid\left(\mathcal{B}^{\gamma}\right)^{\gamma}\right)
$$

is a closed subset for the pseudo-metric defined by $\delta(A, B)=\pi(A \triangle B)$, and contains the algebra $\bigcup_{N \in \mathbf{N}} \sigma\left(\gamma_{-N}^{N}\right)$ by lemma 30 applied once to $(\alpha, \gamma)$ and once to $(\gamma, \gamma)$. Therefore, these collection equals the whole $\sigma$-field $\mathcal{Z}$ itself. In particular, $H\left(\left\{A, A^{c}\right\} \mid \mathcal{B}^{\gamma}\right)=0$ whenever $A \in \mathcal{B}^{\gamma} \vee \mathcal{B}^{\alpha}$ or $A \in\left(\mathcal{B}^{\gamma}\right)^{\gamma}$. Hence $\mathcal{B}^{\gamma} \vee \mathcal{B}^{\alpha}$ and $\left(\mathcal{B}^{\gamma}\right)^{\gamma}$ are contained in $\mathcal{B}^{\gamma}$ modulo the null sets. The result follows.

We now achieve the proof of proposition 18 Assume that $T$ has finite entropy and that $\gamma$ is a generator of $T$. We have to prove that $\mathcal{B}^{\prime} \subset \overline{\mathcal{B}^{\gamma}}$. So let $A \in \mathcal{B}^{\prime}$ and $\alpha=\left\{A, A^{c}\right\}$. For every $n \geq 0$, set

$$
\mathcal{D}_{n}:=\sigma\left(\bigvee_{k \geq n} T^{-k} \alpha\right)
$$

Since, $\mathcal{D}_{1}=\alpha_{1}^{\infty}$, the equality $H\left(\alpha \mid \alpha_{1}^{\infty} \vee \mathcal{B}\right)=h(T, \alpha \mid \mathcal{B})=0$ yields $\alpha \subset \mathcal{D}_{1} \vee \mathcal{B} \bmod \pi$, so $\mathcal{D}_{0}=\mathcal{D}_{1} \vee \mathcal{B} \bmod \pi$. By applying $T^{-n}$, we get more generally $\mathcal{D}_{n}=\mathcal{D}_{n+1} \vee \mathcal{B}$ $\bmod \pi$. By induction, $\mathcal{D}_{0}=\mathcal{D}_{n} \vee \mathcal{B} \bmod \pi$ for every $n \geq 0$. Hence $\mathcal{D}_{0}=\mathcal{B}^{\alpha} \subset \mathcal{B}^{\gamma}$ $\bmod \pi$, thanks to the last corollary, so $A \in \overline{\mathcal{B}^{\gamma}}$. We are done.

\subsection{Proof of proposition 20}

Fix a $d$-dimensional Brownian motion $B$ generating the filtration $\mathcal{B}$ modulo the null sets.

Let $t>\varepsilon>0$. Since $\mathcal{B}$ is immersed in $\mathcal{Z}$, the Brownian motion $B^{(\varepsilon)}:=B_{\varepsilon+}-B_{\varepsilon}$ is independent of $\mathcal{Z}_{\varepsilon}$, which is the terminal $\sigma$-field of the filtration $\left(\mathcal{B}_{\varepsilon} \vee \mathcal{Z}_{s}\right)_{s \in[0, \varepsilon]}$. Therefore

$$
\begin{aligned}
\mathcal{B}_{t}^{\prime} & =\bigcap_{s \in] 0, \varepsilon]}\left(\sigma\left(\left(B_{r}^{(\varepsilon)}\right)_{r \in[0, t-\varepsilon]}\right) \vee \mathcal{B}_{\varepsilon} \vee \mathcal{Z}_{s}\right) \\
& =\sigma\left(\left(B_{r}^{(\varepsilon)}\right)_{r \in[0, t-\varepsilon]}\right) \vee \bigcap_{s \in] 0, \varepsilon]}\left(\mathcal{B}_{\varepsilon} \vee \mathcal{Z}_{s}\right) \\
& =\sigma\left(\left(B_{r}^{(\varepsilon)}\right)_{r \in[0, t-\varepsilon]}\right) \vee \mathcal{B}_{\varepsilon}^{\prime} \bmod \mathbb{P} .
\end{aligned}
$$

Hence, the filtration $\mathcal{B}^{\prime}$ has independent increments after $\varepsilon$, provided by the increments of $B$ after $\varepsilon$, so $\mathcal{B}^{\prime}$ is Brownian after 0 .

Moreover, since $B^{(\varepsilon)}$ is independent of $\mathcal{Z}_{\varepsilon}$, the equality modulo $\mathbb{P}$ above shows that for every $t>\varepsilon>0, \mathcal{B}_{t}^{\prime}$ and $\mathcal{Z}_{\varepsilon}$ are independent conditionally on $\mathcal{B}_{\varepsilon}^{\prime}$. This conditional independence still holds when $\varepsilon=0$, since $\mathcal{Z}_{0}$ is trivial. Thus $\mathcal{B}^{\prime}$ is immersed in $\mathcal{Z}$. By proposition $\left[\right.$ (or corollary 1 in [11]), $\mathcal{B}^{\prime}$ is a $d$-dimensional Brownian filtration.

For every $t>0, \mathcal{B}_{t} \subset \mathcal{B}_{t}^{\prime} \subset \mathcal{B}_{t} \vee \mathcal{Z}_{t}=\mathcal{Z}_{t} \bmod \pi$. These inclusions modulo $\mathbb{P}$ still hold when $t=0$ since $\mathcal{B}_{0}$ and $\mathcal{Z}_{0+}=\bigcap_{s>0} \mathcal{Z}_{s}$ are trivial. Since $\mathcal{B}$ is immersed in $\mathcal{Z}$, we deduce that $\mathcal{B}$ is immersed in $\mathcal{B}^{\prime}$.

Last, let $t \geq 0$. For every $n \geq 1$,

$$
\mathcal{B}_{t}^{\prime \prime} \subset \overline{\mathcal{B}_{t}^{\prime} \vee \mathcal{Z}_{1 / n}} \subset \overline{\overline{\left(\mathcal{B}_{t} \vee \mathcal{Z}_{1 / n}\right)} \vee \mathcal{Z}_{1 / n}}=\overline{\mathcal{B}_{t} \vee \mathcal{Z}_{1 / n}}
$$

If $A \in \mathcal{B}_{t}^{\prime \prime}$, then for each $n \geq 1$, one can find $B_{n} \in \mathcal{B}_{t} \vee \mathcal{Z}_{1 / n}$ such that $\mathbb{P}\left(A \triangle B_{n}\right)=0$; hence $A \in \mathcal{B}_{t}^{\prime}$ since $B:=\lim \sup _{n} B_{n}$ belongs to $\bigcap_{n \geq 1}\left(\mathcal{B}_{t} \vee \mathcal{Z}_{1 / n}\right)$ and $\mathbb{P}(A \triangle B)=0$. The equality $\mathcal{B}_{t}^{\prime \prime}=\mathcal{B}_{t}^{\prime}$ follows. 


\subsection{Proof of the statements of example 21}

Assume that $\mathcal{X}$ is maximal in $\mathcal{Z}$. Since $\mathcal{B}$ is immersed $\mathcal{B}^{\prime}$, we have only to check the inclusion $\mathcal{B}_{\infty}^{\prime} \subset \mathcal{B}_{\infty} \bmod \mathbb{P}$. The maximality of $\mathcal{X}$ in $\mathcal{Z}$ yields

$$
\mathcal{B}_{\infty}^{\prime} \subset \bigcap_{s>0}\left(\mathcal{X}_{\infty} \vee \mathcal{Z}_{s}\right)=\mathcal{X}_{\infty} \bmod \mathbb{P}
$$

so one only needs to check that $\mathbb{E}\left[h(X) \mid \mathcal{B}_{\infty}^{\prime}\right]=\mathbb{E}\left[h(X) \mid \mathcal{B}_{\infty}\right]$ almost surely for every real bounded measurable functional $h$ defined on the space $\mathcal{C}\left(\mathbb{R}_{+}\right)$of all continous functions from $\mathbb{R}_{+}$to $\mathbb{R}$. Since the topology of uniform convergence on compact subsets on the space $\mathcal{C}\left(\mathbb{R}_{+}\right)$is metrizable, it is sufficient to check the equality when $h$ is continuous. In this case, the random variable $h(X)$ is the limit in $L^{1}(\mathbb{P})$ of the sequence $\left(h\left(X^{(n)}\right)\right)_{n \geq 1}$, where $T_{n}$ denotes the first zero of $X$ after time $1 / n$, and $X_{t}^{(n)}=X_{T_{n}+t}$ for every $t \geq 0$. Since $B$ generates the same filtration as $|X|$ up to null sets, $\mathcal{B}_{\infty} \vee \mathcal{Z}_{T_{n}}=\sigma\left(\left|X^{(n)}\right|\right) \vee \mathcal{Z}_{T_{n}}$ $\bmod \mathbb{P}$. But $X^{(n)}$ is independent of $\mathcal{Z}_{T_{n}}$ since $\mathcal{X}$ is immersed in $\mathcal{Z}$, so

$$
\mathbb{E}\left[h\left(X^{(n)}\right) \mid \mathcal{B}_{\infty} \vee \mathcal{Z}_{T_{n}}\right]=\mathbb{E}\left[h\left(X^{(n)}\right) \mid \sigma\left(\left|X^{(n)}\right|\right) \vee \mathcal{Z}_{T_{n}}\right]=\mathbb{E}\left[h\left(X^{(n)}\right) \mid \sigma\left(\left|X^{(n)}\right|\right)\right] \text { a.s.. }
$$

But $\sigma\left(\left|X^{(n)}\right|\right) \subset \mathcal{B}_{\infty} \subset \mathcal{B}_{\infty}^{\prime} \subset \mathcal{B}_{\infty} \vee \mathcal{Z}_{1 / n} \subset \mathcal{B}_{\infty} \vee \mathcal{Z}_{T_{n}}$, so

$$
\mathbb{E}\left[h\left(X^{(n)}\right) \mid \mathcal{B}_{\infty}^{\prime}\right]=\mathbb{E}\left[h\left(X^{(n)}\right) \mid \mathcal{B}_{\infty}\right] .
$$

The statements follow.

\section{Complementability implies maximality: proofs}

\subsection{Key lemma for factors of a Lebesgue automorphism}

Proposition 23 follows from the next lemma, which can derived from lemma in 21] or from lemma 2.3 in 3 .

Lemma 32. Let $\mathcal{A}, \mathcal{B}, \mathcal{C}$ be three factors of $T$. Assume that:

1. $\mathcal{A} \supset \mathcal{B}$;

2. $h(T, \mathcal{A})=h(T, \mathcal{B})<+\infty$;

3. $(T, \mathcal{C})$ is a K-automorphism and has finite entropy.

4. $\mathcal{B}$ and $\mathcal{C}$ are independent.

Then $\mathcal{A}$ and $\mathcal{C}$ are independent.

First, we show how to deduce proposition 23 from lemma 32

Proof. (Proof of proposition 23) Let $\mathcal{C}$ be an independent complement of $\mathcal{B}$ having the property $K$. Let $\mathcal{A}$ be a factor of $T$ such that $\mathcal{A} \supset \mathcal{B}$ and $h(T, \mathcal{A})=h(T, \mathcal{B})$. Then lemma 32 yields that $\mathcal{A}$ and $\mathcal{C}$ are independent. But $\mathcal{Z}=\mathcal{B} \vee \mathcal{C} \subset \mathcal{A} \vee \mathcal{C} \subset \mathcal{Z}$, so $\mathcal{A} \vee \mathcal{C}=\mathcal{B} \vee \mathcal{C}$. Hence $\mathcal{A}=\mathcal{B}$ by the next lemma.

We have just used the follwing general statement, which will also help us in the context of Brownian filtrations. 
Lemma 33. Let $\mathcal{A}, \mathcal{B}, \mathcal{C}$ be three sub- $\sigma$-fields of any probability space $(Z, \mathcal{Z}, \pi)$ such that

- $\mathcal{A} \supset \mathcal{B}$

- $\mathcal{A}$ and $\mathcal{C}$ are independent;

- $\mathcal{A} \vee \mathcal{C}=\mathcal{B} \vee \mathcal{C}$

Then $\mathcal{A}=\mathcal{B} \bmod \pi$.

Proof. Let $A \in \mathcal{A}$. Then $\sigma(A) \vee \mathcal{B} \subset \mathcal{A}$, so $\sigma(A) \vee \mathcal{B}$ is independent of $\mathcal{C}$, and

$$
\pi[A \mid \mathcal{B}]=\pi[A \mid \mathcal{B} \vee \mathcal{C}]=\pi[A \mid \mathcal{A} \vee \mathcal{C}]=\mathbf{1}_{A} \pi \text {-almost surely }
$$

Hence $A \in \mathcal{B} \bmod \pi$.

We now give two different proofs of lemma 32 The second one relies on Pinsker's formula and is a bit simpler.

\subsection{Proof of lemma 32}

The proof below is a reformulation of the proof given in 21.

Assume that the assumptions hold. Let $\alpha, \beta, \gamma$ be countable partitions generating $(T, \mathcal{A}),(T, \mathcal{B}),(T, \mathcal{C})$, respectively. Since $(T, \mathcal{A}),(T, \mathcal{B}),(T, \mathcal{C})$ have finite entropy, $\alpha$, $\beta, \gamma$ have also finite entropy. Given $n \geq 1$, set $\alpha_{0}^{n-1}=\alpha \vee \cdots \vee T^{-(n-1)} \alpha, \beta_{0}^{n-1}=$ $\beta \vee \cdots \vee T^{-(n-1)} \beta$,

$$
\mathcal{C}_{n}=\sigma\left(\bigvee_{q \in \mathbf{Z}} T^{-q n} \gamma\right) \text { and } \mathcal{D}_{n}=\sigma\left(\bigvee_{k \geq n} T^{-k} \gamma\right)
$$

Then $\mathcal{C}_{n}$ is a factor of $T^{n}, \alpha_{0}^{n-1} \vee \gamma$ is a generator of $\left(T^{n}, \mathcal{A} \vee \mathcal{C}_{n}\right)$ whereas $\beta_{0}^{n-1} \vee \gamma$ is a generator of $\left(T^{n}, \mathcal{B} \vee \mathcal{C}_{n}\right)$.

Therefore, on the one hand,

$$
\begin{aligned}
h\left(T^{n}, \mathcal{A} \vee \mathcal{C}_{n}\right) & =H\left(\alpha_{0}^{n-1} \vee \gamma \mid \bigvee_{q \geq 1} T^{-q n}\left(\alpha_{0}^{n-1} \vee \gamma\right)\right) \\
& =H\left(\alpha_{0}^{n-1} \vee \gamma \mid \bigvee_{k \geq n} T^{-k} \alpha \vee \bigvee_{q \geq 1} T^{-q n} \gamma\right) \\
& =H\left(\alpha_{0}^{n-1} \mid \bigvee_{k \geq n} T^{-k} \alpha \vee \bigvee_{q \geq 1} T^{-q n} \gamma\right)+H\left(\gamma \mid \bigvee_{k \geq 0} T^{-k} \alpha \vee \bigvee_{q \geq 1} T^{-q n} \gamma\right) \\
& \leq H\left(\alpha_{0}^{n-1} \mid \bigvee_{k \geq n} T^{-k} \alpha\right)+H(\gamma \mid \alpha) \\
& =h\left(T^{n}, \mathcal{A}\right)+H(\gamma \mid \alpha)=n h(T, \mathcal{A})+H(\gamma \mid \alpha) .
\end{aligned}
$$

On the other hand, by independence of $\mathcal{B}$ and $\mathcal{C}$,

$$
\begin{aligned}
h\left(T^{n}, \mathcal{B} \vee \mathcal{C}_{n}\right) & =H\left(\beta_{0}^{n-1} \mid \bigvee_{k \geq n} T^{-k} \beta \vee \bigvee_{q \geq 1} T^{-q n} \gamma\right)+H\left(\gamma \mid \bigvee_{k \geq 0} T^{-k} \beta \vee \bigvee_{q \geq 1} T^{-q n} \gamma\right) \\
& =H\left(\beta_{0}^{n-1} \mid \bigvee_{k \geq n} T^{-k} \beta\right)+H\left(\gamma \mid \bigvee_{q \geq 1} T^{-q n} \gamma\right) \\
& \geq h\left(T^{n}, \mathcal{B}\right)+H\left(\gamma \mid \mathcal{D}_{n}\right)=n h(T, \mathcal{B})+H\left(\gamma \mid \mathcal{D}_{n}\right)
\end{aligned}
$$


But $h\left(T^{n}, \mathcal{B} \vee \mathcal{C}_{n}\right) \leq h\left(T^{n}, \mathcal{A} \vee \mathcal{C}_{n}\right)$ since $\mathcal{B} \subset \mathcal{A}$. Putting things together and using the assumption $h(T, \mathcal{A})=h(T, \mathcal{B})<+\infty$ yields $H\left(\gamma \mid \mathcal{D}_{n}\right) \leq H(\gamma \mid \alpha)$.

But $\left(\mathcal{D}_{n}\right)_{n \geq 1}$ is a decreasing sequence of $\sigma$-fields with trivial intersection since $(T, \mathcal{C})$ has the property $K$, so $H\left(\gamma \mid \mathcal{D}_{n}\right) \rightarrow H(\gamma)$ as $n \rightarrow+\infty$. Hence, $H(\gamma) \leq H(\gamma \mid \alpha)$, so $\alpha$ and $\gamma$ are independent. This conclusion is preserved if one replaces the generators $\alpha$ and $\gamma$ by the supremum of $T^{-k} \alpha$ and $T^{-k} \gamma$ over all $k \in \llbracket-n, n \rrbracket$. Letting $n$ go to infinity yields the independence of $\mathcal{A}$ and $\mathcal{C}$.

\subsection{Alternative proof of lemma 32}

The inclusion $\mathcal{A} \supset \mathcal{B}$ and the independence of $\mathcal{B}$ and $\mathcal{C}$ yield

$$
h(T, \mathcal{A})+h(T, \mathcal{C}) \geq h(T, \mathcal{A} \vee \mathcal{C}) \geq h(T, \mathcal{B} \vee \mathcal{C})=h(T, \mathcal{B})+h(T, \mathcal{C})
$$

But $h(T, \mathcal{A})=h(T, \mathcal{B})$, hence $h(T, \mathcal{A} \vee \mathcal{C})=h(T, \mathcal{A})+h(T, \mathcal{C})$. Since $(T, \mathcal{C})$ is a $K$ automorphism with finite entropy, Berg's lemma below shows that $\mathcal{A}$ and $\mathcal{C}$ independent.

Lemma 34. (lemma 2.3 in [3]) Let $\mathcal{A}$ and $\mathcal{C}$ be two factors of the dynamical system $(Z, \mathcal{Z}, \pi, T)$, such that $h(T, \mathcal{A} \vee \mathcal{C})=h(T, \mathcal{A})+h(T, \mathcal{C})<+\infty$ and $(T, \mathcal{C})$ is a $K$ automorphism. Then $\mathcal{A}$ and $\mathcal{C}$ independent.

Proof. Let $\alpha$ and $\gamma$ be countable generating partitions of $(T, \mathcal{A})$ and $(T, \mathcal{C})$, respectively. Set

$$
\alpha_{1}^{\infty}=\bigvee_{k \geq 1} T^{-k} \alpha \text { and } \gamma_{1}^{\infty}=\bigvee_{k \geq 1} T^{-k} \gamma
$$

Then $h(T, \mathcal{A})=H\left(\alpha \mid \alpha_{1}^{\infty}\right), h(T, \mathcal{C})=H\left(\gamma \mid \gamma_{1}^{\infty}\right)$ and $h(T, \mathcal{A} \vee \mathcal{C})=H\left(\alpha \vee \gamma \mid \alpha_{1}^{\infty} \vee \gamma_{1}^{\infty}\right)$ But Pinsker's formula (proposition 77 in section 7 or theorem 6.3 in [22]) gives

$$
H\left(\alpha \vee \gamma \mid \alpha_{1}^{\infty} \vee \gamma_{1}^{\infty}\right)=H\left(\alpha \mid \alpha_{1}^{\infty}\right)+H\left(\gamma \mid \mathcal{A} \vee \gamma_{1}^{\infty}\right)
$$

So the assumption $h(T, \mathcal{A} \vee \mathcal{C})=h(T, \mathcal{A})+h(T, \mathcal{C})<+\infty$ yields $H\left(\gamma \mid \gamma_{1}^{\infty}\right)=H\left(\gamma \mid \mathcal{A} \vee \gamma_{1}^{\infty}\right)$

For any partition $\delta \subset \mathcal{A}$ with finite entropy, we derive $H\left(\gamma \mid \delta \vee \gamma_{1}^{\infty}\right)=H\left(\gamma \mid \gamma_{1}^{\infty}\right)$, so

$$
H\left(\delta \vee \gamma \mid \gamma_{1}^{\infty}\right)=H\left(\delta \mid \gamma_{1}^{\infty}\right)+H\left(\gamma \mid \delta \vee \gamma_{1}^{\infty}\right)=H\left(\delta \mid \gamma_{1}^{\infty}\right)+H\left(\gamma \mid \gamma_{1}^{\infty}\right)
$$

But we have also

$$
H\left(\delta \vee \gamma \mid \gamma_{1}^{\infty}\right)=H\left(\gamma \mid \gamma_{1}^{\infty}\right)+H\left(\delta \mid \gamma \vee \gamma_{1}^{\infty}\right)
$$

Hence $H\left(\delta \mid \gamma_{1}^{\infty}\right)=H\left(\delta \mid \gamma \vee \gamma_{1}^{\infty}\right)$.

Let $m \geq 0$ and $n$ be integers. Applying the last equality to $\delta:=\bigvee_{|k| \leq m} T^{n-k} \alpha$ yields

$$
H\left(\bigvee_{|k| \leq m} T^{n-k} \alpha \mid \bigvee_{k \geq 1} T^{-k} \gamma\right)=H\left(\bigvee_{|k| \leq m} T^{n-k} \alpha \mid \bigvee_{k \geq 0} T^{-k} \gamma\right)
$$

Since $T$ preserves $\pi$, this is equivalent to

$$
H\left(\bigvee_{|k| \leq m} T^{-k} \alpha \mid \bigvee_{k \geq n+1} T^{-k} \gamma\right)=H\left(\bigvee_{|k| \leq m} T^{-k} \alpha \mid \bigvee_{k \geq n} T^{-k} \gamma\right)
$$

As a result, the entropy above does not depend on $n$. Letting $n$ go to $-\infty$ and to $+\infty$, and using the fact that $(T, \mathcal{C})$ is a $K$-automorphism, we get at the limit

$$
H\left(\bigvee_{|k| \leq m} T^{-k} \alpha \mid \mathcal{C}\right)=H\left(\bigvee_{|k| \leq m} T^{-k} \alpha\right)
$$

so the partition $\bigvee_{|k| \leq m} T^{-k} \alpha$ is independent of $\mathcal{C}$. Letting $m$ go to $+\infty$ yields the independence of $\mathcal{A}$ and $\mathcal{C}$. 


\subsection{Proof in the context of Brownian filtrations}

The proof of proposition 25 below may look suspiciously simple, but actually, it relies on non-trivial theorems of stochastic integration, namely the predictable representation property and the bracket characterization of multi-dimensional Brownian motions among local martingales. The immersion of a filtration into another one is a strong property, as shown for example by the characterizations for a Brownian filtration recalled in the introduction (proposition 2). The key step is very similar to lemma 32,

Lemma 35. Let A, B, C be three Brownian motions in some filtration $\mathcal{Z}$. Assume that:

1. $\sigma(A) \supset \sigma(B)$;

2. $A$ and $B$ have the same finite dimension;

3. $B$ and $C$ are independent.

Then $A$ and $C$ are independent.

Proof. Call $p$ the dimension of $A$ and $B$ and $q$ the (possibly infinite) dimension of $C$. Since $B$ is a Brownian motion in $\mathcal{Z}$ and its own filtration, it is also a Brownian motion in the intermediate filtration $\mathcal{F}^{A}$. Hence, one can find an $\mathcal{F}^{A}$-predictable process $H$ with values in the group of all orthogonal $p \times p$ matrices such that

$$
B=\int_{0}^{\cdot} H_{s} \mathrm{~d} A_{s} .
$$

Since $H_{s}^{\top} H_{s}=I_{p}$ for every $s \geq 0$ (where $H_{s}^{\top}$ denotes the transpose of $H_{s}$ ), we have also

$$
A=\int_{0}^{\cdot} H_{s}^{\top} \mathrm{d} B_{s} .
$$

Looking at the components, we get for every $i \in \llbracket 1, p \rrbracket$,

$$
A^{(i)}=\sum_{j=1}^{p} \int_{0}^{\cdot} H_{s}(j, i) \mathrm{d} B_{s}^{(j)} .
$$

For every $i \in \llbracket 1, p \rrbracket$ and $k \in \llbracket 1, q \rrbracket$, we get

$$
\left\langle A^{(i)}, C^{(k)}\right\rangle=\sum_{j=1}^{p} \int_{0}^{.} H_{s}(j, i) \mathrm{d}\left\langle B^{(j)}, C^{(k)}\right\rangle_{s}=0,
$$

since $\left\langle B^{(j)}, C^{(k)}\right\rangle=0$ by independence of $B$ et $C$. We derive that $(A, C)$ is a $p+q$ dimensional Brownian motion in $\mathcal{Z}$, so $A$ and $C$ are independent.

Deducing proposition 25 from the last lemma involves almost the same arguments as deducing proposition 23 from lemma 32 .

Proof. Proof of proposition 25. Let $\mathcal{Z}$ be a finite Brownian filtration, and $\mathcal{A}, \mathcal{B}, \mathcal{C}$ be three Brownian filtrations in $\mathcal{Z}$ such that $\mathcal{A}_{t} \supset \mathcal{B}_{t}$ for every $t \geq 0, \mathcal{A}$ and $\mathcal{B}$ have the same dimension, and $\mathcal{C}$ is an independent complement of $\mathcal{B}$ in $\mathcal{Z}$.

Let $A, B, C$ be Brownian motions generating $\mathcal{A}, \mathcal{B}, \mathcal{C}$ modulo the null events. Then lemma 35 applies, so $A$ is independent of $C$. Then lemma 33 applies, so $\sigma(A)=\sigma(B)$ $\bmod \mathbb{P}$. But $\mathcal{F}^{B}$ is immersed in $\mathcal{F}^{A}$. Since the final $\sigma$-fields $\mathcal{F}_{\infty}^{A}=\sigma(A)$ and $\mathcal{F}_{\infty}^{B}=\sigma(B)$ coincide almost surely, we get $\mathcal{F}_{t}^{A}=\mathcal{F}_{t}^{B} \bmod \mathbb{P}$ for every $t \geq 0$ by lemma 1 . 
We now prove proposition 27 .

Proof. Let $\mathcal{C}$ be a complement of $\mathcal{B}$ after 0 , and $\mathcal{A}$ be a $d$-dimensional Brownian filtration immersed in $\mathcal{Z}$ and containing $\mathcal{B}$. Let $A, B, C$ be Brownian motions in $\mathcal{Z}$ generating $\mathcal{A}, \mathcal{B}, \mathcal{C}$ respectively modulo the null events. Since $\mathcal{A}$ and $\mathcal{B}^{\prime}$ are immersed in $\mathcal{Z}$, it is sufficient to prove the inclusion $\mathcal{A}_{\infty} \subset \mathcal{B}_{\infty}^{\prime}$. Hence, given $s>0$, we have to check that $\mathcal{A}_{\infty} \subset \mathcal{B}_{\infty} \vee \mathcal{Z}_{s} \bmod \mathbb{P}$.

By lemma 35, we know that $A$ and $C$ are independent Brownian motions in $\mathcal{F}^{Z}$. Thus $\mathcal{Z}_{s}, A_{s+}-A_{s}$ and $C_{s+.}-C_{s}$ are independent. Let

$$
\begin{gathered}
\tilde{\mathcal{A}}:=\mathcal{A}_{\infty} \vee \mathcal{Z}_{s}=\sigma\left(A_{s+\cdot}-A_{s}\right) \vee \mathcal{Z}_{s} \bmod \mathbb{P}, \\
\tilde{\mathcal{B}}:=\mathcal{B}_{\infty} \vee \mathcal{Z}_{s}=\sigma\left(B_{s+\cdot}-B_{s}\right) \vee \mathcal{Z}_{s} \bmod \mathbb{P}, \\
\tilde{\mathcal{C}}:=\sigma\left(C_{s+.}-C_{s}\right) .
\end{gathered}
$$

Then $\tilde{\mathcal{A}} \supset \tilde{\mathcal{B}}, \tilde{\mathcal{B}}$ and $\tilde{\mathcal{C}}$ are independent, and $\tilde{\mathcal{A}} \vee \tilde{\mathcal{C}}=\tilde{\mathcal{B}} \vee \tilde{\mathcal{C}} \bmod \mathbb{P}$, since

$$
\mathcal{Z}_{\infty} \supset \tilde{\mathcal{A}} \vee \tilde{\mathcal{C}} \supset \tilde{\mathcal{B}} \vee \tilde{\mathcal{C}}=\mathcal{B}_{\infty} \vee \mathcal{C}_{\infty} \vee \mathcal{Z}_{s}=\mathcal{Z}_{\infty} \bmod \mathbb{P}
$$

Hence lemma 33 applies, so $\mathcal{A}_{\infty} \subset \tilde{\mathcal{A}}=\tilde{\mathcal{B}}=\mathcal{B}_{\infty} \vee \mathcal{Z}_{s} \bmod \mathbb{P}$.

\section{A maximal but not-complementable Brownian filtration}

\subsection{A maximal but not-complementable filtration in a dyadic product- type filtration}

This subsection is devoted to the proof of the following lemma, which is the first step of the construction of a maximal but not-complementable Brownian filtration.

Lemma 36. One can construct :

- a dyadic product-type filtration $\left(\mathcal{Z}_{n}\right)_{n \leq 0}$,

- a poly-adic filtration $\left(\mathcal{U}_{n}\right)_{n \leq 0}$ immersed in $\left(\mathcal{Z}_{n}\right)_{n \leq 0}$,

- a random variable $U$ with values in some Polish space $(E, \mathcal{E})$ and generating $\mathcal{U}_{0}$,

such that for $U(\mathbb{P})$-almost every $u \in E,\left(\mathcal{Z}_{n}\right)_{n \leq 0}$ is Kolmogorovian but not producttype under $\mathbb{P}_{u}=\mathbb{P}[\cdot \mid U=u]$. Therefore, the filtration $\left(\mathcal{U}_{n}\right)_{n \leq 0}$ is maximal but non complementable in $\left(\mathcal{Z}_{n}\right)_{n \leq 0}$.

Proof. We begin with a variant of an example given in [8], which was itself inspired from an unpublished note of Tsirelson [26].

For every $n \leq 0$, call $K_{n}$ the finite field with $q_{n}=2^{2^{|n|}}$ elements. Start with a sequence of independent random variables $\left(Z_{n}\right)_{n \leq 0}$ such that for every $n \leq 0, Z_{2 n}=\left(X_{n}, Y_{n}\right)$ is uniform on $K_{n}^{4} \times K_{n}^{4}$ and $Z_{2 n-1}=B_{n}$ is uniform on $K_{n}^{4}$. By construction, the filtration $\left(\mathcal{F}_{n}^{Z}\right)_{n \leq 0}$ is product-type and $\left(r_{n}\right)_{n \leq 0}$-adic, with $r_{2 n-1}=q_{n}^{4}$ and $r_{2 n}=q_{n}^{8}$ for every $n \leq 0$.

Since $\left|K_{n-1}\right|=2^{2^{|n|+1}}=\left|K_{n}\right|^{2}$, one can fix a bijection between $K_{n-1}^{4} \times K_{n-1}^{4}$ and the set $\mathcal{M}_{4}\left(K_{n}\right)$ of all $4 \times 4$ matrices with entries in $K_{n}$. Call $A_{n}$ the uniform random 
variable on $\mathcal{M}_{4}\left(K_{n}\right)$ corresponding to $Z_{2 n-2}$ through this bijection, and set $U_{2 n-1}=0$ and $U_{2 n}=Y_{n}-A_{n} X_{n}-B_{n}$.

For every $n \leq 0,\left(X_{n}, Y_{n}\right)$ is independent of $\mathcal{F}_{2 n-1}^{Z}$ and uniform on $K_{n}^{4} \times K_{n}^{4}$. Since the random map $(x, y) \mapsto\left(x, y-A_{n} x-B_{n}\right)$ from $K_{n}^{4} \times K_{n}^{4}$ to itself is $\mathcal{F}_{2 n-1}^{Z}$-measurable and bijective, $\left(X_{n}, U_{2 n}\right)$ is also independent of $\mathcal{F}_{2 n-1}^{Z}$ and uniform on $K_{n}^{4} \times K_{n}^{4}$ and is still an innovation at time $2 n$ of the filtration $\mathcal{F}^{Z}$. Therefore, the filtration $\left(\mathcal{F}_{n}^{U}\right)_{n \leq 0}$ is immersed in $\left(\mathcal{F}_{n}^{Z}\right)_{n \leq 0}$, product-type and $\left(r_{n} / q_{n}^{4}\right)_{n \leq 0}$-adic.

As the integers $\left(r_{n}\right)_{n<0}$ and $\left(r_{n} / q_{n}^{4}\right)_{n<0}$ are powers of 2 , one can interpolate the filtrations $\left(\mathcal{F}_{n}^{Z}\right)_{n \leq 0}$ and $\left(\mathcal{F}_{n}^{U}\right)_{n \leq 0}$ to get two filtrations $\mathcal{Z}=\left(\mathcal{Z}_{n}\right)_{n \leq 0}$ and $\mathcal{U}=\left(\mathcal{U}_{n}\right)_{n \leq 0}$ such that:

- $\mathcal{Z}$ is a dyadic product-type filtration,

- $\mathcal{U}$ is poly-adic and immersed in $\mathcal{Z}$,

- $\left(\mathcal{F}_{n}^{Z}\right)_{n \leq 0}=\left(\mathcal{Z}_{t_{n}}\right)_{n \leq 0}$ and $\left(\mathcal{F}_{n}^{U}\right)_{n \leq 0}=\left(\mathcal{U}_{t_{n}}\right)_{n \leq 0}$ for some sequence $0=t_{0}>t_{-1}>$ $t_{-2}>\ldots$ of integers.

To get such filtrations, it suffices to split the random variables $\left(B_{n}\right)_{n \leq 0},\left(X_{n}\right)_{n \leq 0}$ and $\left(U_{2 n}\right)_{n \leq 0}$ into independent random bits. The bits coming from the $B_{n}$ and the $X_{n}$ provide innovations of the filtration $\mathcal{Z}$ at the times when the filtration $\mathcal{Z}$ only increases. The bits coming from the $U_{2 n}$ provide innovations of the two filtrations $\mathcal{Z}$ and $\mathcal{U}$ at the times when both of them increase.

The random variable $U=\left(U_{n}\right)_{n \leq 0}$ generates $\mathcal{U}_{0}=\mathcal{F}_{0}^{U}$. Let us check that for $U(\mathbb{P})$ almost every $u \in E,\left(\mathcal{Z}_{n}\right)$ is Kolmogorovian but not product-type under $\mathbb{P}_{u}=\mathbb{P}[\cdot \mid U=u]$. By corollary 11 and proposition 22 of the present paper (propositions 3,4 and corollary 9 of [18]), the last two statements will follow.

First, we note that for every $n \leq 0, \mathcal{F}_{2 n}^{Z}=\mathcal{F}_{2 n}^{U} \vee \mathcal{F}_{n}^{B, X}$, where $\mathcal{F}_{2 n}^{U}$ and $\mathcal{F}_{n}^{B, X}$ are independent. By corollary 11, for $U(\mathbb{P})$-almost every $u \in E$, the filtration $\left(\mathcal{F}_{2 n}^{Z}\right)_{n \leq 0}$ seen under $\mathbb{P}_{u}$ is isomorphic to the filtration $\left(\mathcal{F}_{n}^{B, X}\right)_{n \leq 0}$ seen under $\mathbb{P}$, which is product-type. Thus, $\mathcal{Z}_{-\infty}=\mathcal{F}_{-\infty}^{Z}$ is trivial under $\mathbb{P}_{u}$.

To show that $\left(\mathcal{Z}_{n}\right)$ is not product-type under $\mathbb{P}_{u}$, it suffices to show that the extracted filtration $\left(\mathcal{F}_{n}^{Z}\right)_{n \leq 0}$ is not product-type under $\mathbb{P}_{u}$. To do this, we check that the random variable $Z_{0}$ does not satisfy the I-cosiness criterion. Let $Z^{\prime}=\left(X^{\prime}, Y^{\prime}\right)$ and $Z^{\prime \prime}=\left(X^{\prime \prime}, Y^{\prime \prime}\right)$ be two copies of the process $Z$ under $\mathbb{P}_{u}$, defined on some probability space $\left(\bar{\Omega}, \overline{\mathcal{A}}, \overline{\mathbb{P}}_{u}\right)$, such that both natural filtrations $\mathcal{F}^{Z^{\prime}}$ and $\mathcal{F}^{Z^{\prime \prime}}$ are immersed in some filtration $\mathcal{G}$.

For every $n \leq 0$, define the copies $A_{n}^{\prime}, A_{n}^{\prime \prime}$ and $B_{n}^{\prime}, B_{n}^{\prime \prime}$ of the random variables $A_{n}$ and $B_{n}$ by the obvious way, and set $S_{n}=\left\{x \in K_{n}^{4}: A_{n}^{\prime} x+B_{n}^{\prime}=A_{n}^{\prime \prime} x+B_{n}^{\prime \prime}\right\}$. Then for $U(\mathbb{P})$ almost every $u \in E$, the equalities $Y_{n}^{\prime}=A_{n}^{\prime} X_{n}^{\prime}+B_{n}^{\prime}+u_{2 n}$ and $Y_{n}^{\prime \prime}=A_{n}^{\prime \prime} X_{n}^{\prime \prime}+B_{n}^{\prime \prime}+u_{2 n}$ hold $\overline{\mathbb{P}}_{u}$-almost surely. Therefore,

$$
\mathbf{1}_{\left[Z_{2 n}^{\prime}=Z_{2 n}^{\prime \prime}\right]}=\mathbf{1}_{\left[X_{n}^{\prime}=X_{n}^{\prime \prime} \in S_{n}\right]} \leq \mathbf{1}_{\left[X_{n}^{\prime} \in S_{n}\right]} \overline{\mathbb{P}}_{u^{-} \text {-almost surely }}
$$

But the random variable $S_{n}$ is $\mathcal{G}_{2 n-1}$-measurable whereas $X_{n}^{\prime}$ is uniform on $K_{n}^{4}$ conditionally on $\mathcal{G}_{2 n-1}$ since $\mathcal{F}^{Z^{\prime}}$ is immersed in $\mathcal{G}$. Thus

$$
\overline{\mathbb{P}}_{u}\left[Z_{2 n}^{\prime}=Z_{2 n}^{\prime \prime} \mid \mathcal{G}_{2 n-1}\right] \leq \frac{\left|S_{n}\right|}{q_{n}^{4}} \leq \mathbf{1}_{\left[A_{n}^{\prime}=A_{n}^{\prime \prime}\right]}+\frac{1}{q_{n}} \mathbf{1}_{\left[A_{n}^{\prime} \neq A_{n}^{\prime \prime}\right]} \overline{\mathbb{P}}_{u^{-}} \text {almost surely, }
$$


since $S_{n}$ contains at most $q_{n}^{3}$ points when $A_{n}^{\prime} \neq A_{n}^{\prime \prime}$. Passing to the complements and taking the expectations yields

$$
\overline{\mathbb{P}}_{u}\left[Z_{2 n}^{\prime} \neq Z_{2 n}^{\prime \prime}\right] \geq\left(1-\frac{1}{q_{n}}\right) \overline{\mathbb{P}}_{u}\left[A_{n}^{\prime} \neq A_{n}^{\prime \prime}\right]=\left(1-\frac{1}{q_{n}}\right) \overline{\mathbb{P}}_{u}\left[Z_{2 n-2}^{\prime} \neq Z_{2 n-2}^{\prime \prime}\right] .
$$

By induction, one gets that for every $n \leq 0$

$$
\overline{\mathbb{P}}_{u}\left[Z_{0}^{\prime} \neq Z_{0}^{\prime \prime}\right] \geq \prod_{k=n+1}^{0}\left(1-\frac{1}{q_{k}}\right) \times \overline{\mathbb{P}}_{u}\left[Z_{2 n}^{\prime} \neq Z_{2 n}^{\prime \prime}\right]
$$

If, for some $N>-\infty$, the $\sigma$-fields $\mathcal{F}_{N}^{Z^{\prime}}$ and $\mathcal{F}_{N}^{Z^{\prime \prime}}$ are independent, then $\overline{\mathbb{P}}_{u}\left[Z_{2 n}^{\prime} \neq Z_{2 n}^{\prime \prime}\right] \rightarrow$ 1 as $n \rightarrow-\infty$, so

$$
\overline{\mathbb{P}}_{u}\left[Z_{0}^{\prime} \neq Z_{0}^{\prime \prime}\right] \geq \prod_{k \leq 0}\left(1-\frac{1}{q_{k}}\right)>0
$$

The proof is complete.

\subsection{Embedding dyadic filtrations in Brownian filtrations}

We start with the two filtrations provided by lemma 36. By construction, the filtration $\left(\mathcal{Z}_{n}\right)_{n \leq 0}$ can be generated by some i.i.d. sequence $\left(\varepsilon_{n}\right)_{n \leq 0}$ of uniform random variables with values in $\{-1,1\}$.

The filtration $\left(\mathcal{U}_{n}\right)_{n \leq 0}$ is $\left(a_{n}\right)_{n \leq 0}$-adic for some sequence $\left(a_{n}\right)_{n \leq 0}$ taking values 1 and 2 only. Call $D \subset \mathbf{Z}_{-}$the set of all $n \leq 0$ such that $a_{n}=2$. Since $\left(\mathcal{U}_{n}\right)_{n \leq 0}$ is poly-adic and immersed in a product-type filtration, $\left(\mathcal{U}_{n}\right)_{n \leq 0}$ is also product-type. Therefore, the filtration $\left(\mathcal{U}_{n}\right)_{n \leq 0}$ can be generated by some sequence $\left(\eta_{n}\right)_{n \leq 0}$ of independent random variables with $\eta_{n}$ uniform on $\{-1,1\}$ if $n \in D, \eta_{n}=0$ if $n \notin D$.

By immersion of $\left(\mathcal{U}_{n}\right)_{n \leq 0}$ in $\left(\mathcal{Z}_{n}\right)_{n \leq 0}$, each $\eta_{n}$ is $\mathcal{Z}_{n}$-measurable and independent of $\mathcal{Z}_{n-1}$. So when $n \in D, \eta_{n}$ can be written $\eta_{n}=H_{n} \varepsilon_{n}$, where $H_{n}$ is some $\mathcal{Z}_{n-1}$-random variable taking values in $\{-1,1\}$.

Fix an increasing sequence $\left(t_{n}\right)_{n \leq 0}$ of positive real numbers such that $t_{0}=1$ and $t_{n} \rightarrow 0$ as $n \rightarrow-\infty$ (e.g. $t_{n}=2^{n}$ for every $n \leq 0$ ). By symmetry and independence of Brownian increments, one may construct a Brownian motion $X$ such that for every $n \leq 0, \varepsilon_{n}=\operatorname{sign}\left(X_{t_{n}}-X_{t_{n-1}}\right)$. Let $Y$ be another Brownian motion, independent of $X$.

Since $\mathcal{Z}_{n-1} \subset \mathcal{F}_{t_{n-1}}^{X, Y}$ for every $n \leq 0$, one gets a predictable process $\left(A_{t}\right)_{0<t \leq 1}$ with values in $O_{2}(\mathbb{R})$ and two independent Brownian motions $B$ and $C$ in $\mathcal{F}^{X, Y}$ on the timeinterval $[0,1]$ by setting for every $\left.t \in] t_{n-1}, t_{n}\right]$,

$$
A_{t}=\left(\begin{array}{cc}
H_{n} & 0 \\
0 & 1
\end{array}\right) \text { if } n \in D, \quad A_{t}=\left(\begin{array}{ll}
0 & 1 \\
1 & 0
\end{array}\right) \text { if } n \notin D,
$$

and for every $t>0$,

$$
\left(\begin{array}{c}
d B_{t} \\
d C_{t}
\end{array}\right)=A_{t}\left(\begin{array}{c}
d X_{t} \\
d Y_{t}
\end{array}\right)
$$

Theorem 37. The filtration generated by the Brownian motion $B$ thus defined is complementable after 0 , maximal, but not complementable in $\mathcal{F}^{X, Y}$. 


\section{Proof. Complementability after 0}

Let us check that $C$ is a complement after 0 of $B$, or equivalently that

$$
\forall s \in] 0,1], \mathcal{F}_{1}^{B, C} \vee \mathcal{F}_{s}^{X, Y}=\mathcal{F}_{1}^{X, Y} .
$$

Since $t_{0}=1$ and $t_{m} \rightarrow 0+$ as $m \rightarrow-\infty$, it is sufficient to consider instants $s$ which are some subdivision time $t_{m}$ with $m \leq 0$. Since for every $n \geq m$, the process $A$ coincides on each time-interval $\left.] t_{n}, t_{n+1}\right]$ with an $\mathcal{F}_{t_{n}}^{X, Y}$-measurable random variable, the formula

$$
\left(\begin{array}{c}
d X_{t} \\
d Y_{t}
\end{array}\right)=A_{t}^{-1}\left(\begin{array}{c}
d B_{t} \\
d C_{t}
\end{array}\right)
$$

enables us to recover $(X, Y)$ from the knowledge of $\left(\left(X_{s}, Y_{s}\right)\right)_{0 \leq s \leq t_{m}}$ and $(B, C)$.

\section{Maximality}

By proposition 27, the maximality of $B$ will follow from its complementability after 0 once we will have proved the equality

$$
\mathcal{F}_{1}^{B}=\bigcap_{s \in] 0,1]}\left(\mathcal{F}_{1}^{B} \vee \mathcal{F}_{s}^{X, Y}\right)
$$

The intersection above, over all $s \in] 0,1]$ can be restricted to the instants $t_{m}$ with $m \leq 0$.

It is now convenient to introduce the notations

$$
\Delta X_{n}=\left(X_{t}-X_{t_{n-1}}\right)_{t_{n-1} \leq t \leq t_{n}} \text { and } \varepsilon_{n} \Delta X_{n}=\left(\varepsilon_{n}\left(X_{t}-X_{t_{n-1}}\right)\right)_{t_{n-1} \leq t \leq t_{n}} .
$$

Recall that $\varepsilon_{n}=\operatorname{sign}\left(X_{t_{n}}-X_{t_{n-1}}\right)$. Therefore, $\sigma\left(\Delta X_{n}\right)=\sigma\left(\varepsilon_{n}\right) \vee \sigma\left(\varepsilon_{n} \Delta X_{n}\right)$, with $\sigma\left(\varepsilon_{n}\right)$ and $\sigma\left(\varepsilon_{n} \Delta X_{n}\right)$ independent by symmetry of Brownian increments.

We define in the same way the random variables $\Delta Y_{n}, \Delta B_{n}, \Delta C_{n}$ and $\eta_{n} \Delta B_{n}$. Then

$$
\begin{gathered}
\Delta B_{n}=H_{n} \Delta X_{n}=\eta_{n} \varepsilon_{n} \Delta X_{n} \text { and } \Delta C_{n}=\Delta Y_{n} \text { if } n \in D, \\
\Delta B_{n}=\Delta Y_{n} \text { and } \Delta C_{n}=\Delta X_{n} \text { if } n \in D^{c} .
\end{gathered}
$$

Moreover, when $n \in D, \eta_{n}=\operatorname{sign}\left(B_{t_{n}}-B_{t_{n-1}}\right)$ is independent of $\eta_{n} \Delta B_{n}=\varepsilon_{n} \Delta X_{n}$.

Therefore, $\mathcal{F}_{1}^{B}=\mathcal{A} \vee \mathcal{B}$, with

$$
\mathcal{A}=\sigma\left(\left(\eta_{n}\right)_{n \in D}\right), \quad \mathcal{B}=\sigma\left(\left(\varepsilon_{n} \Delta X_{n}\right)_{n \in D}\right) \vee \sigma\left(\left(\Delta Y_{n}\right)_{n \in D^{c}}\right) .
$$

For $n \in \mathbf{Z}_{-}$, set $\left.\left.\left.\left.D_{n}=D \cap\right]-\infty, n\right], D_{n}^{c}=\right]-\infty, n\right] \backslash D$, and

$$
\mathcal{C}_{n}=\mathcal{F}_{n}^{\varepsilon}, \quad \mathcal{D}_{n}=\sigma\left(\left(\varepsilon_{k} \Delta X_{k}\right)_{k \in D_{n}^{c}}\right) \vee \sigma\left(\left(\Delta Y_{k}\right)_{k \in D_{n}}\right) .
$$

Then $\mathcal{F}_{n}^{\varepsilon} \vee \mathcal{F}_{t_{n}}^{C}=\mathcal{C}_{n} \vee \mathcal{D}_{n}$.

The maximality of $\mathcal{F}^{\eta}$ in $\mathcal{F}^{\varepsilon}$ yields the equality

$$
\mathcal{A}=\bigcap_{n \leq 0}\left(\mathcal{A} \vee \mathcal{C}_{n}\right) \quad \bmod \mathbb{P} .
$$

By independence of $B$ and $C$, the $\sigma$-fields $\mathcal{B}$ and $\mathcal{D}_{0}$ are independent, so corollary 39 applies and the following exchange property holds

$$
\mathcal{B}=\mathcal{B} \vee \mathcal{D}_{-\infty}=\bigcap_{n \leq 0}\left(\mathcal{B} \vee \mathcal{D}_{n}\right) \quad \bmod \mathbb{P}
$$


Since the three sequences $\left(\varepsilon_{n}\right)_{n \leq 0},\left(\varepsilon_{n} \Delta X_{n}\right)_{n \leq 0}$ and $\left(\Delta Y_{n}\right)_{n \leq 0}$ are independent, the $\sigma$ fields $\mathcal{A} \vee \mathcal{C}_{0}=\mathcal{F}_{0}^{\varepsilon}$ and $\mathcal{B} \vee \mathcal{D}_{0}=\mathcal{F}_{0}^{\varepsilon \Delta X, Y}$ are independent. Hence, lemma 38 yields

$\mathcal{F}_{1}^{B}=\mathcal{A} \vee \mathcal{B}=\bigcap_{n \leq 0}\left(\mathcal{A} \vee \mathcal{B} \vee \mathcal{C}_{n} \vee \mathcal{D}_{n}\right)=\bigcap_{n \leq 0}\left(\mathcal{F}_{1}^{B} \vee \mathcal{F}_{n}^{\varepsilon} \vee \mathcal{F}_{t_{n}}^{C}\right)=\bigcap_{n \leq 0}\left(\mathcal{F}_{1}^{B} \vee \mathcal{F}_{t_{n}}^{X, Y}\right) \bmod \mathbb{P}$

This proves the maximality of $B$.

\section{Non-complementability}

Keep the notations introduced in the proof of the maximality and set $\varepsilon:=\left(\varepsilon_{n}\right)_{n \leq 0}$, $\eta:=\left(\eta_{n}\right)_{n \leq 0}$. Remind that $\varepsilon,\left(\varepsilon_{n} \Delta X_{n}\right)_{n \leq 0}$ and $\left(\Delta Y_{n}\right)_{n \leq 0}$ are independent families of independent random variables and that $\mathcal{F}_{1}^{B}$ is the $\sigma$-field generated by $\eta,\left(\varepsilon_{n} \Delta X_{n}\right)_{n \in D}$ and $\left(\Delta Y_{n}\right)_{n \in D^{c}}$.

The filtration $\left(\mathcal{F}_{t_{n}}^{X, Y}\right)_{n \leq 0}$ can be splitted into three independent parts, namely

$$
\mathcal{F}_{t_{n}}^{X, Y}=\mathcal{F}_{n}^{\varepsilon} \vee \sigma\left(\left(\varepsilon_{k} \Delta X_{k}\right)_{k \in D_{n}} \cup\left(\Delta Y_{k}\right)_{k \in D_{n}^{c}}\right) \vee \sigma\left(\left(\Delta Y_{k}\right)_{k \in D_{n}} \cup\left(\varepsilon_{k} \Delta X_{k}\right)_{k \in D_{n}^{c}}\right) .
$$

The second part is a function of $B$ whereas the third part is independent of $(\varepsilon, B)$. By independent enlargement, we get that for $B(\mathbb{P})$-almost every $b \in C([0,1], \mathbb{R})$, the filtration $\left(\mathcal{F}_{n}^{\varepsilon}\right)_{n \leq 0}$ is immersed in $\left(\mathcal{F}_{t_{n}}^{X, Y}\right)_{n \leq 0}$ under $\mathbb{P}[\cdot \mid B=b]$.

But $\eta$ is some measurable function $\Phi$ of $B$ and is also a function of $\varepsilon$. Since $\varepsilon$, $\left(\varepsilon_{n} \Delta X_{n}\right)_{n \in D}$ and $\left(\Delta Y_{n}\right)_{n \in D^{c}}$ are independent, the law of $\varepsilon$ under $\mathbb{P}[\cdot \mid B=b]$ coincides with the law of $\varepsilon$ under $\mathbb{P}[\cdot \mid \eta=\Phi(b)]$.

Since $\eta$ generates the same $\sigma$-field as the random variable $U$ of lemma 36, we derive that for $B(\mathbb{P})$-almost every $b \in C([0,1], \mathbb{R})$, the filtration $\left(\mathcal{F}_{n}^{\varepsilon}\right)_{n \leq 0}$ is $\left(2 / a_{n}\right)$-adic but not product-type under $\mathbb{P}[\cdot \mid B=b]$. But this filtration is immersed in $\left(\mathcal{F}_{t_{n}}^{X, Y}\right)_{n \leq 0}$ under $\mathbb{P}[\cdot \mid B=b]$, hence by Vershik's theorem (theorem 5 in the present paper), $\left(\mathcal{F}_{t_{n}}^{X, Y}\right)_{n \leq 0}$ cannot be product-type so $\mathcal{F}^{X, Y}$ cannot be Brownian under $\mathbb{P}[\cdot \mid B=b]$. Thus, the Brownian filtration $\mathcal{F}^{B}$ is not complementable in $\mathcal{F}^{X, Y}$.

Lemma 38. Let $\mathcal{A}, \mathcal{B}$ be two sub- $\sigma$-fields and $\left(\mathcal{C}_{n}\right)_{n \leq 0},\left(\mathcal{D}_{n}\right)_{n \leq 0}$ be two filtrations of the probability space $(\Omega, \mathcal{T}, \mathbb{P})$. If

$$
\mathcal{A}=\bigcap_{n \leq 0}\left(\mathcal{A} \vee \mathcal{C}_{n}\right) \quad \bmod \mathbb{P}, \quad \mathcal{B}=\bigcap_{n \leq 0}\left(\mathcal{B} \vee \mathcal{D}_{n}\right) \quad \bmod \mathbb{P},
$$

and if $\mathcal{A} \vee \mathcal{C}_{0}$ and $\mathcal{B} \vee \mathcal{D}_{0}$ are independent, then

$$
\mathcal{A} \vee \mathcal{B}=\bigcap_{n \leq 0}\left(\mathcal{A} \vee \mathcal{B} \vee \mathcal{C}_{n} \vee \mathcal{D}_{n}\right) \quad \bmod \mathbb{P}
$$

Proof. Since both sides of the equality to be proved are sub- $\sigma$-fields of $\mathcal{A} \vee \mathcal{B} \vee \mathcal{C}_{0} \vee \mathcal{D}_{0}$, it is sufficient to prove that for every $Z \in L^{1}\left(\mathcal{A} \vee \mathcal{C}_{0} \vee \mathcal{B} \vee \mathcal{D}_{0}\right)$, one has

$$
\mathbb{E}[Z \mid \mathcal{A} \vee \mathcal{B}]=\mathbb{E}\left[Z \mid \bigcap_{n \leq 0}\left(\mathcal{A} \vee \mathcal{B} \vee \mathcal{C}_{n} \vee \mathcal{D}_{n}\right)\right]
$$

Considering only random variables $Z=X Y$ with $X \in L^{1}\left(\mathcal{A} \vee \mathcal{C}_{0}\right)$ and $Y \in L^{1}\left(\mathcal{B} \vee \mathcal{D}_{0}\right)$ is sufficient since these random variables span a dense subspace in $L^{1}\left(\mathcal{A} \vee \mathcal{C}_{0} \vee \mathcal{B} \vee \mathcal{D}_{0}\right)$. Given $Z$ as above, one has $\mathbb{E}\left[Z \mid \mathcal{A} \vee \mathcal{C}_{0} \vee \mathcal{B}\right]=X \mathbb{E}[Y \mid \mathcal{B}]$, so

$$
\mathbb{E}[Z \mid \mathcal{A} \vee \mathcal{B}]=\mathbb{E}[X \mid \mathcal{A} \vee \mathcal{B}] \mathbb{E}[Y \mid \mathcal{B}]=\mathbb{E}[X \mid \mathcal{A}] \mathbb{E}[Y \mid \mathcal{B}]
$$


since $\sigma(X) \vee \mathcal{A}$ is independent of $\mathcal{B}$. In the same way, one gets that for every $n \leq 0$,

$$
\mathbb{E}\left[Z \mid \mathcal{A} \vee \mathcal{B} \vee \mathcal{C}_{n} \vee \mathcal{D}_{n}\right]=\mathbb{E}\left[X \mid \mathcal{A} \vee \mathcal{C}_{n}\right] \mathbb{E}\left[Y \mid \mathcal{B} \vee \mathcal{D}_{n}\right]
$$

Thus, taking the limit as $n \rightarrow-\infty$ yields the result by the martingale convergence theorem and the assumption.

The particular case where $\mathcal{B}$ and the $\sigma$-fields $\mathcal{C}_{n}$ are equal to $\{\emptyset, \Omega\}$ yields the following classical and useful result.

Corollary 39. Let $\mathcal{A}$ be a sub- $\sigma$-field and $\left(\mathcal{D}_{n}\right)_{n \leq 0}$ be a filtration of the probability space $(\Omega, \mathcal{T}, \mathbb{P})$. If $\mathcal{A}$ and $\mathcal{D}_{0}$ are independent, then

$$
\mathcal{A}=\bigcap_{n \leq 0}\left(\mathcal{A} \vee \mathcal{D}_{n}\right) \quad \bmod \mathbb{P}
$$

\section{A complementable factor arising from a non-complemen- table filtration}

\subsection{Definition of a uniform randomised decimation process}

We denote by $\{a, b\}^{\infty}$ the set of all infinite words on the alphabet $\{a, b\}$, namely the set of all maps from $\mathbf{N}=\{1,2, \ldots\}$ to $\{a, b\}$. We endow this set with the uniform probability measure $\mu$ : a random infinite word $X$ is chosen according to $\mu$ if the successive letters $X(1), X(2), \ldots$ form a sequence of independent and uniform random variables taking values in $\{a, b\}$.

We denote by $\mathcal{P}(\mathbf{N})$ the power set of $\mathbf{N}$, i.e. the set of all subsets of $\mathbf{N}$. Given $p \in] 0,1[$, we define the probability measure $\nu_{p}$ on $\mathcal{P}(\mathbf{N})$ as follows: the law of a random subset $I$ of $\mathbf{N}$ is $\nu_{p}$ if $\mathbf{1}_{I}(1), \mathbf{1}_{I}(2), \ldots$ form an i.i.d. sequence of Bernoulli random variables with parameter $p$. Equivalently, this means that $\mathbb{P}[F \subset I]=p^{|F|}$ for every finite subset $F$ of $\mathbf{N}$. In this case, we note that almost surely, $I$ is infinite with infinite complement. The law $\nu:=\nu_{1 / 2}$ will be called the uniform law on $\mathcal{P}(\mathbf{N})$.

When $A$ is an infinite subset of $\mathbf{N}$, we denote by $\psi_{A}(1)<\psi_{A}(2)<\ldots$ its elements. This defines an increasing map $\psi_{A}$ from $\mathbf{N}$ to $\mathbf{N}$ whose range is $A$. Conversely, for every increasing map $f$ from $\mathbf{N}$ to $\mathbf{N}$, there is a unique infinite subset $A$ of $\mathbf{N}$, namely the range of $f$, such that $f=\psi_{A}$. These remarks lead to the following statement.

Lemma 40. Let $I$ and $J$ be independent random infinite subsets of $\mathbf{N}$ with respective laws $\nu_{p}$ and $\nu_{q}$, and $R=\psi_{I} \circ \psi_{J}(\mathbf{N})=\psi_{I}(J)$ be the range of $\psi_{I} \circ \psi_{J}$. Then $\psi_{I} \circ \psi_{J}=\psi_{R}$ and the law of $R$ is $\nu_{p q}$.

Proof. The equality $\psi_{I} \circ \psi_{J}=\psi_{R}$ follows from the remarks above. Let $F$ be a finite subset of $\mathbf{N}$. By injectivity of $\psi_{I}$,

$$
[F \subset R]=\left[F \subset I ; \psi_{I}^{-1}(F) \subset J\right]
$$

and $[F \subset I]=\left[\left|\psi_{I}^{-1}(F)\right|=|F|\right]$, therefore by independence of $I$ and $J$,

$$
\mathbb{P}[F \subset R \mid \sigma(I)]=\mathbf{1}_{[F \subset I]} \mathbb{P}\left[\psi_{I}^{-1}(F) \subset J \mid \sigma(I)\right]=\mathbf{1}_{[F \subset I]} q^{\left|\psi_{I}^{-1}(F)\right|}=\mathbf{1}_{[F \subset I]} q^{|F|} .
$$

Thus $\mathbb{P}[F \subset R]=\mathbb{P}[F \subset I] q^{|F|}=(p q)^{|F|}$. 
Here is another property that we will use to define the uniform randomised decimation process on $\{a, b\}$, and also later, in the proof of proposition 50 .

Lemma 41. Let $X$ be a uniform random word on $\{a, b\}^{\infty}$. Let I be a random subset of $\mathbf{N}$ with law $\nu_{p}$, independent of $X$. Then

- $I, X \circ \psi_{I}, X \circ \psi_{I^{c}}$ are independent

- $X \circ \psi_{I}, X \circ \psi_{I^{c}}$ are uniform random words on $\{a, b\}^{\infty}$.

Proof. Almost surely, $I$ is infinite with infinite complement, so the random maps $\psi_{I}$ and $\psi_{I^{c}}$ are well-defined. The integers $\psi_{I}(1), \psi_{I^{c}}(1), \psi_{I}(2), \psi_{I^{c}}(2) \ldots$ are distinct, so conditionally on $I$, the random variables $X\left(\psi_{I}(1)\right), X\left(\psi_{I^{c}}(1)\right), X\left(\psi_{I}(2)\right), X\left(\psi_{I^{c}}(2)\right), \ldots$ are independent and uniform on $\{a, b\}$. The result follows.

Definition 42. Call $\mathcal{P}^{\prime}(\mathbf{N})$ the set of all infinite subsets of $\mathbf{N}$. A uniform randomised decimation process in the alphabet $\{a, b\}$ is a stationary Markow chain $\left(X_{n}, I_{n}\right)_{n \in \mathbf{Z}}$ with values in $\{a, b\}^{\infty} \times \mathcal{P}^{\prime}(\mathbf{N})$ defined as follows: for every $n \in \mathbf{Z}$,

1. the law of $\left(X_{n}, I_{n}\right)$ is $\mu \otimes \nu$;

2. $I_{n}$ is independent of $\left(X_{n-1}, I_{n-1}\right)$ and uniform on $\mathcal{P}(\mathbf{N})$;

3. $X_{n}=X_{n-1} \circ \psi_{I_{n}}$.

Such a process is well-defined and unique in law since the law $\mu \otimes \nu$ is invariant by the transition kernel given by conditions 2 and 3 above, thanks to lemma 41 Moreover, $\left(I_{n}\right)_{n \in \mathbf{Z}}$ is a sequence of innovations for the filtration $\mathcal{F}^{X, I}$. Therefore, the filtration $\mathcal{F}^{X, I}$ has independent increments or is locally of product-type, according to Laurent's terminology [16].

This process is a kind of randomisation of Vershik's decimation process given in example 3 of [27. Indeed, Vershik's decimation process is equivalent to the process that we would get by choosing the random sets $I_{n}$ uniformly among the set of all even positive integers and the set of all odd positive integers. Although Vershik's decimation process generates a non-standard filtration, we will show that our randomised process generates a standard one.

Theorem 43. The uniform randomised decimation process on the alphabet $\{a, b\}$ generates a product-type filtration.

\subsection{Proof of theorem 43}

We have seen that the filtration $\mathcal{F}^{X, I}$ admits $\left(I_{n}\right)_{n \in \mathbf{Z}}$ as a sequence of innovations. Each innovation has diffuse law. Therefore, to prove that the filtration $\left(\mathcal{F}_{n}^{X, I}\right)_{n \leq 0}$, or equivalently, the filtration $\left(\mathcal{F}_{n}^{X, I}\right)_{n \in \mathbf{Z}}$ is product-type, it suffices to check Vershik's first level criterion (see reminders further and definition 2.6 and theorem 2.25 in [16]). Concretely, we have to check any random variable in $L^{1}\left(\mathcal{F}_{0}^{X, I}, \mathbb{R}\right)$ can be approached in $L^{1}\left(\mathcal{F}_{0}^{X, I}, \mathbb{R}\right)$ by measurable functions of finitely many innovations of $\left(\mathcal{F}^{X, I}\right)_{n \leq 0}$.

The innovations $\left(I_{n}\right)_{n \in \mathbf{Z}}$ are inadequate to do this, since the random variable $X_{0}$ is independent of the whole sequence $\left(I_{n}\right)_{n \in \mathbf{Z}}$, so functions of the $\left(I_{n}\right)_{n \in \mathbf{Z}}$ cannot approach non-trivial functions of $X_{0}$. Therefore, we will have to construct new innovations. The next lemma gives us a general procedure to get some. 
Lemma 44. Fix $n \in \mathbf{Z}$. Let $\Phi$ be some $\mathcal{F}_{n-1}^{X, I}$-measurable map from $\mathbf{N}$ to $\mathbf{N}$. If $\Phi$ is almost surely bijective, then the random variable $J_{n}=\Phi\left(I_{n}\right)$ is independent of $\mathcal{F}_{n-1}^{X, I}$ and uniform on $\mathcal{P}(\mathbf{N})$.

Proof. For every finite subset $F$ of $\mathbf{N}$,

$$
P\left[F \subset J_{n} \mid \mathcal{F}_{n-1}^{X, I}\right]=P\left[\Phi^{-1}(F) \subset I_{n} \mid \mathcal{F}_{n-1}^{X, I}\right]=(1 / 2)^{\left|\Phi^{-1}(F)\right|}=(1 / 2)^{|F|} \text { almost surely. }
$$

The result follows.

Actually, the proof of theorem 43 is similar to the proof of the standardness of the erased-words filtration by S. Laurent [17] and uses the same tools, namely canonical coupling and cascaded permutations.

\section{Definition 45. (Canonical word and canonical coupling)}

The infinite canonical word $C$ on the alphabet $\{a, b\}$ is the word abab $\cdots$, namely the map from $\mathbf{N}$ to $\{a, b\}$ which sends the odd integers on $a$ and the even integers on $b$.

If $x$ is an infinite word $x$ on the alphabet $\{a, b\}$, namely a map from $\mathbf{N}$ to $\{a, b\}$, we set for every $i \in \mathbf{N}$,

$$
\begin{array}{r}
\phi_{x}(i)=2 q-1 \text { if } x(i) \text { is the } q \text {-th occurence of the letter a in } x, \\
\phi_{x}(i)=2 q \text { if } x(i) \text { is the } q \text {-th occurence of the letter } b \text { in } x .
\end{array}
$$

Lemma 46. By definition, the map $\phi_{x}$ thus defined from $\mathbf{N}$ to $\mathbf{N}$ is injective and satisfies the equality $x=C \circ \phi_{x}$. When each possible letter $a$ or $b$ appears infinitely many times in $x, \phi_{x}$ is a permutation of $\mathbf{N}$, (called canonical coupling by S. Laurent).

Roughly speaking, if $x$ is a typical word of $\{a, b\}^{\infty}$ endowed with the uniform law, the asymptotic proportions of $a$ and $b$ are $1 / 2$ are $1 / 2$, so $\phi_{x}$ is asymptotically close to the identity map.

\section{Definition 47. (New innovations and cascaded permutations)}

Let $\Omega^{\prime}$ be the almost sure event on which

- each possible letter a or $b$ appears infinitely many times in the infinite word $X_{0}$;

- each subset $I_{n}$ is infinite.

On $\Omega^{\prime}$, we define by recursion a sequence $\left(\Phi_{n}\right)_{n \geq 0}$ of random permutations of $\mathbf{N}$ and a sequence $\left(J_{n}\right)_{n \geq 1}$ of random infinite subsets of $\mathbf{N}$ by setting $\Phi_{0}=\phi_{X_{0}}$ and, for every $n \geq 1$,

$$
J_{n}=\Phi_{n-1}\left(I_{n}\right) \text { and } \Phi_{n-1} \circ \psi_{I_{n}}=\psi_{J_{n}} \circ \Phi_{n} .
$$

Let us check that the inductive construction above actually works $\Omega^{\prime}$.

On $\Omega^{\prime}$, the map $\Phi_{0}=\phi_{X_{0}}$ is bijective by lemma 46,

Once we know that $\Phi_{n-1}$ is a random permutations of $\mathbf{N}$, the map $\Phi_{n-1} \circ \psi_{I_{n}}$ is a random injective map from $\mathbf{N}$ to $\mathbf{N}$ with range $\Phi_{n-1}\left(I_{n}\right)=J_{n}$. Therefore, $J_{n}$ is infinite and the map $\Phi_{n}$ is well defined by equation 1 for every $k \in \mathbf{N}, \Phi_{n}(k)$ is the rank of the integer $\Phi_{n-1}\left(\psi_{I_{n}}(k)\right)$ in the set $J_{n}$. Moreover, $\Phi_{n}$ is a permutation of $\mathbf{N}$. 
Informally, the cascaded permutations $\left(\Phi_{n}\right)_{n \geq 0}$ are induced by $\Phi_{0}=\phi_{X_{0}}$ and the successive extractions. More precisely, equation 1 is represented by a commutative diagramm which gives the correspondance between the positions of a same letter in different words.

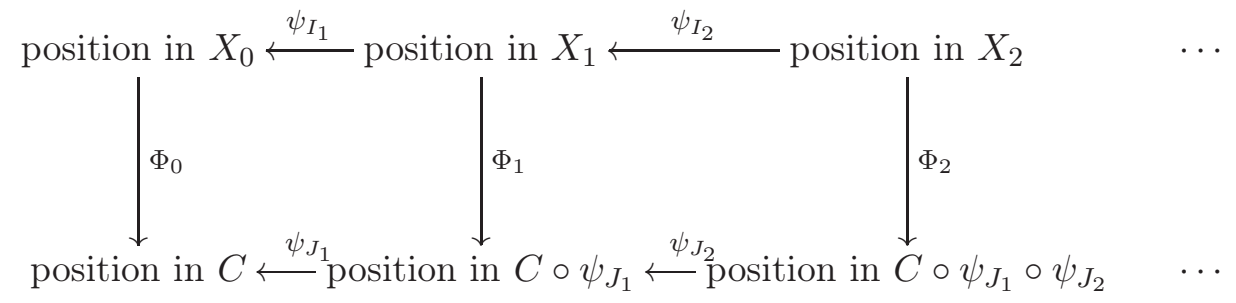

Here is a realisation of the first three steps. The boldface numbers form the subsets $I_{1}, J_{1}, I_{2}, J_{2}, \ldots$. Among the arrows representing $\phi_{X_{0}}$, the plain arrows (from elements in $I_{1}$ to elements in $J_{1}$ ) provide the permutation $\phi_{X_{0}, I_{1}}$ by renumbering of the elements.
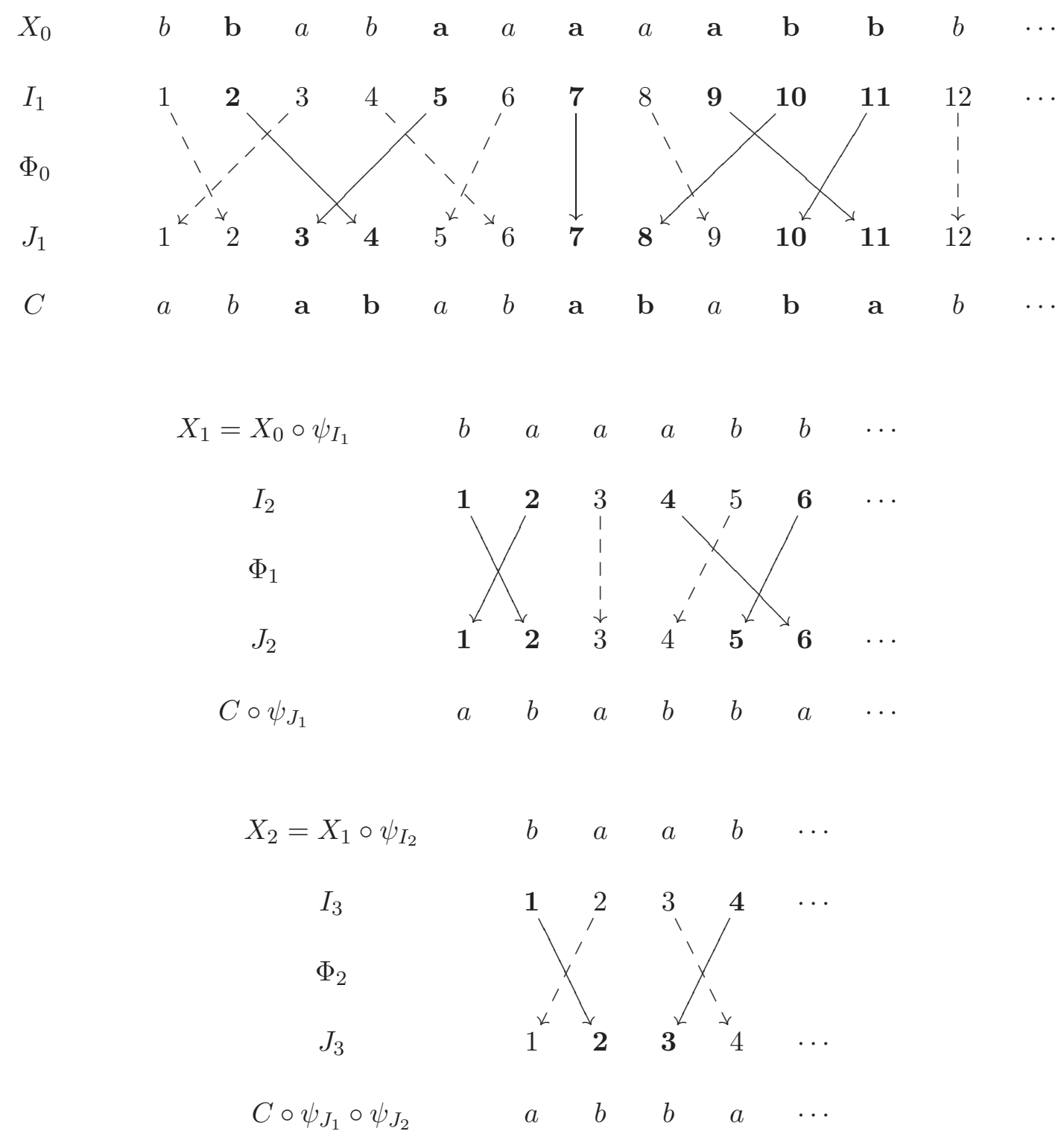
Lemma 48. On the almost sure event $\Omega^{\prime}$, the following properties hold for every $n \geq 1$,

1. $J_{n}$ is independent of $\mathcal{F}_{n-1}^{X, I}$ and is uniform on $\mathcal{P}(\mathbf{N})$.

2. $\sigma\left(X_{0}, J_{1}, \ldots, J_{n}\right)=\sigma\left(X_{0}, I_{1}, \ldots, I_{n}\right)$.

3. the random map $\Phi_{n}$ is $\sigma\left(X_{0}, J_{1}, \ldots, J_{n}\right)$-measurable.

4. $\phi_{X_{0}} \circ \psi_{I_{1}} \circ \cdots \circ \psi_{I_{n}}=\psi_{J_{1}} \circ \cdots \circ \psi_{J_{n}} \circ \Phi_{n}$.

5. $X_{n}=C \circ \psi_{J_{1}} \circ \cdots \circ \psi_{J_{n}} \circ \Phi_{n}$.

Proof. Since $\Phi_{0}=\phi_{X_{0}}$, properties 2, 3, 4, 5 above hold with $n$ replaced by 0 .

Let $n \geq 1$. Assume that properties 2, 3, 4, 5 hold with $n$ replaced by $n-1$.

Then by lemma 44, property 1 holds.

By definition and by the induction hypothesis, the random set $J_{n}=\Phi_{n-1}\left(I_{n}\right)$ is $\sigma\left(X_{0}, I_{1}, \ldots, I_{n}\right)$-measurable. Conversely, since $I_{n}=\Phi_{n-1}^{-1}\left(J_{n}\right)$, the knowledge of $\Phi_{n-1}$ and $J_{n}$ is sufficient to recover $I_{n}$, so property 2 holds.

For every $k \in \mathbf{N}, \Phi_{n}(k)$ is the rank of the integer $\Phi_{n-1}\left(\psi_{I_{n}}(k)\right)$ in the set $\Phi_{n-1}\left(I_{n}\right)$. Thus the random map $\Phi_{n}$ is a measurable for $\sigma\left(X_{0}, J_{1}, \ldots, J_{n-1}, I_{n}\right)=\sigma\left(X_{0}, J_{1}, \ldots, J_{n}\right)$. Therefore, property 3 holds.

By induction hypothesis and by formula 1,

$$
\begin{aligned}
\phi_{X_{0}} \circ \psi_{I_{1}} \circ \cdots \circ \psi_{I_{n}} & =\left(\phi_{X_{0}} \circ \psi_{I_{1}} \circ \cdots \circ \psi_{I_{n-1}}\right) \circ \psi_{I_{n}} \\
& =\left(\psi_{J_{1}} \circ \cdots \circ \psi_{J_{n-1}} \circ \Phi_{n-1}\right) \circ \psi_{I_{n}} \\
& =\left(\psi_{J_{1}} \circ \cdots \circ \psi_{J_{n-1}}\right) \circ\left(\Phi_{n-1} \circ \psi_{I_{n}}\right) \\
& =\left(\psi_{J_{1}} \circ \cdots \circ \psi_{J_{n-1}}\right) \circ\left(\psi_{J_{n}} \circ \Phi_{n}\right) \\
& =\psi_{J_{1}} \circ \cdots \circ \psi_{J_{n}} \circ \Phi_{n},
\end{aligned}
$$

so

$$
\begin{aligned}
X_{n} & =X_{0} \circ \psi_{I_{1}} \circ \cdots \circ \psi_{I_{n}} \\
& =C \circ \phi_{X_{0}} \circ \psi_{I_{1}} \circ \cdots \circ \psi_{I_{n}} \\
& =C \circ \psi_{J_{1}} \circ \cdots \circ \psi_{J_{n}} \circ \Phi_{n},
\end{aligned}
$$

which yields properties 4 and 5 .

Lemma 47 follows by recursion.

The next result shows that the innovations $\left(J_{n}\right)_{n \geq 1}$ constructed above provide better and better approximations of $X_{n}$ as $n \rightarrow+\infty$.

Lemma 49. Fix $L \in \mathbf{N}$. Then $\mathbb{P}\left[X_{n}=C \circ \psi_{J_{1}} \circ \cdots \circ \psi_{J_{n}}\right.$ on $\left.\llbracket 1, L \rrbracket\right] \rightarrow 1$ as $n \rightarrow+\infty$.

Proof. By equality 5 , it suffices to check that, $\mathbb{P}\left(E_{n}\right) \rightarrow 1$ as $n \rightarrow+\infty$, where $E_{n}$ is the event " $\Phi_{n}$ coincides on $\llbracket 1, L \rrbracket$ with the identity map".

By lemma 40, $\psi_{I_{1}} \circ \cdots \circ \psi_{I_{n}}=\psi_{A_{n}}$ and $\psi_{J_{1}} \circ \cdots \circ \psi_{J_{n}}=\psi_{B_{n}}$, where $A_{n}$ and $B_{n}$ are random subsets of $\mathbf{N}$ with law $\nu_{p_{n}}$, where $p_{n}=2^{-n}$.

Therefore, by property 4 of lemma 48, $\phi_{X_{0}} \circ \psi_{A_{n}}=\psi_{B_{n}} \circ \Phi_{n}$, so for each $k \in \mathbf{N}$, $\Phi_{n}(k)$ is the rank of the integer $\phi_{X_{0}}\left(\psi_{A_{n}}(k)\right)$ in the set $\phi_{X_{0}}\left(A_{n}\right)=B_{n}$. 
Thus, the event $E_{n}$ holds if and only if the $L$ first elements of the set $\phi_{X_{0}}\left(A_{n}\right)$ in increasing order are $\phi_{X_{0}}\left(\psi_{A_{n}}(1)\right), \ldots, \phi_{X_{0}}\left(\psi_{A_{n}}(L)\right)$.

Set $\tau_{n, k}=\psi_{A_{n}}(k)$ for every $k \in \mathbf{N}$. Since the law of $A_{n}$ is $\nu_{p_{n}}$, the random variables $\tau_{n, 1}, \tau_{n, 2}-\tau_{n, 1}, \tau_{n, 3}-\tau_{n, 2}, \ldots$ are independent and geometric with parameter $p_{n}$.

We have noted that

$$
E_{n}=\left[\forall k \geq L+1, \phi_{X_{0}}\left(\tau_{n, 1}\right)<\ldots<\phi_{X_{0}}\left(\tau_{n, L}\right)<\phi_{X_{0}}\left(\tau_{n, k}\right)\right]
$$

Roughly speaking, the probability of this event tends to 1 because $\phi_{X_{0}}$ is close to the identity map and the set $A_{n}$ gets sparser and sparser as $n$ increases to infinity. Let us formalize this argument.

Since $X_{0}$ is uniform on $\{a, b\}^{\infty}$, the random variables $\left(\eta_{i}\right)_{i \geq 1}=\left(\mathbf{1}_{\left[X_{0}(i)=b\right]}\right)_{i \geq 1}$ form an i.i.d. sequence of Bernoulli random variables with parameter $1 / 2$. For every $t \in \mathbf{N}$, the random variable $S_{t}=\eta_{1}+\cdots+\eta_{t}$ counts the number of $b$ in the subword $X_{0}(\llbracket 1, t \rrbracket)$, whereas $t-S_{t}$ counts the number of $a$ in the subword $X_{0}(\llbracket 1, t \rrbracket)$, so by definition of $\phi_{X_{0}}$,

$$
\begin{aligned}
\phi_{X_{0}}(t)=2\left(t-S_{t}\right)-1 & \text { if } X_{0}(t)=a \\
\phi_{X_{0}}(t)=2 S_{t} & \text { if } X_{0}(t)=b .
\end{aligned}
$$

Given $t_{1}<t_{2}$ in $\mathbf{N}$, the inequality $\max \left(S_{t_{1}}, t_{1}-S_{t_{1}}\right)<\min \left(S_{t_{2}}, t_{2}-S_{t_{2}}\right)$ implies $\phi_{X_{0}}\left(t_{1}\right)<\phi_{X_{0}}(t)$ for every integer $t \geq t_{2}$. Therefore,

$$
E_{n} \supset\left[\forall k \in \llbracket 1, L \rrbracket, \max \left(S_{\tau_{n, k}}, \tau_{n, k}-S_{\tau_{n, k}}\right)<\min \left(S_{\tau_{n, k+1}}, \tau_{n, k+1}-S_{\tau_{n, k+1}}\right)\right] .
$$

Thus it suffices to prove that for any fixed $k \in \mathbf{N}$,

$$
p_{n, k}:=\mathbb{P}\left[\max \left(S_{\tau_{n, k}}, \tau_{n, k}-S_{\tau_{n, k}}\right) \geq \min \left(S_{\tau_{n, k+1}}, \tau_{n, k+1}-S_{\tau_{n, k+1}}\right)\right] \rightarrow 0 \text { as } n \rightarrow+\infty .
$$

Since $X_{0}$ is independent of $I_{1}, \ldots, I_{n}$, the sequence $\left(S_{t}\right)_{t \geq 1}$ is independent of the sequence $\left(\tau_{n, k}\right)_{k \geq 1}$. Moreover, $\left(S_{t}\right)_{t \geq 1}$ has the same law as $\left(t-S_{t}\right)_{t \geq 1}$ and $S_{\tau_{n, k+1}}-S_{\tau_{n, k}}$ has the same law as $S_{\tau_{n, 1}}$. Therefore, for every integer $x \geq 1$,

$$
\begin{aligned}
p_{n, k} & \leq 2 \mathbb{P}\left[S_{\tau_{n, k+1}} \leq \max \left(S_{\tau_{n, k}}, \tau_{n, k}-S_{\tau_{n, k}}\right)\right] \\
& =2 \mathbb{P}\left[S_{\tau_{n, k+1}}-S_{\tau_{n, k}} \leq \max \left(0, \tau_{n, k}-2 S_{\tau_{n, k}}\right)\right] \\
& \leq 2 \mathbb{P}\left[S_{\tau_{n, k+1}}-S_{\tau_{n, k}} \leq x-1\right]+2 \mathbb{P}\left[\tau_{n, k}-2 S_{\tau_{n, k}} \geq x\right] \\
& =2 \mathbb{P}\left[S_{\tau_{n, 1}} \leq x-1\right]+\mathbb{P}\left[\left|2 S_{\tau_{n, k}}-\tau_{n, k}\right| \geq x\right] .
\end{aligned}
$$

On the one hand, the random variable $S_{\tau_{n, 1}}$ is binomial with parameters $\tau_{n, 1}$ and $1 / 2$ conditionally on $\tau_{n, 1}$, so its generating function is given by

$$
\begin{aligned}
\mathbb{E}\left[z^{S_{\tau_{n}, 1}}\right] & =\mathbb{E}\left[\mathbb{E}\left[z^{S_{\tau_{n}, 1}} \mid \sigma\left(\tau_{n, 1}\right)\right]\right] \\
& =\mathbb{E}\left[\left(\frac{1+z}{2}\right)^{\tau_{n, 1}}\right] \\
& =\frac{p_{n}(1+z) / 2}{1-\left(1-p_{n}\right)(1+z) / 2} \\
& =\frac{p_{n}(1+z)}{1+p_{n}-\left(1-p_{n}\right) z} \\
& =\frac{p_{n}(1+z)}{1+p_{n}} \sum_{m=0}^{+\infty}\left(\frac{1-p_{n}}{1+p_{n}}\right)^{m} z^{m}
\end{aligned}
$$


This yields the law of $S_{\tau_{n, 1}}$, namely

$$
\begin{gathered}
P\left[S_{\tau_{n, 1}}=0\right]=\frac{p_{n}}{1+p_{n}}, \\
P\left[S_{\tau_{n, 1}}=s\right]=\frac{p_{n}}{1+p_{n}}\left(\left(\frac{1-p_{n}}{1+p_{n}}\right)^{s-1}+\left(\frac{1-p_{n}}{1+p_{n}}\right)^{s}\right)=2 p_{n} \frac{\left(1-p_{n}\right)^{s-1}}{\left(1+p_{n}\right)^{s+1}} \text { if } s \geq 1 .
\end{gathered}
$$

Therefore, $\mathbb{P}\left[S_{\tau_{n, 1}}=s\right] \leq 2 p_{n}$ for every $s \geq 0$, so $\mathbb{P}\left[S_{\tau_{n, 1}} \leq x-1\right] \leq 2 p_{n} x$.

On the other hand, $\left(2 S_{t}-t\right)_{t \geq 0}$ is a simple symmetric random walk on $\mathbf{Z}$, independent of $\tau_{n, k}$ so

$$
\mathbb{E}\left[2 S_{\tau_{n, k}}-\tau_{n, k} \mid \sigma\left(\tau_{n, k}\right)\right]=0 \text { and } \operatorname{Var}\left(2 S_{\tau_{n, k}}-\tau_{n, k} \mid \sigma\left(\tau_{n, k}\right)\right)=\tau_{n, k} .
$$

Therefore,

$$
\mathbb{E}\left[2 S_{\tau_{n, k}}-\tau_{n, k}\right]=0 \text { and } \operatorname{Var}\left(2 S_{\tau_{n, k}}-\tau_{n, k}\right)=\operatorname{Var}(0)+\mathbb{E}\left[\tau_{n, k}\right]=k / p_{n},
$$

so Bienaymé-Tchebicheff's inequality yields $\mathbb{P}\left[\left|2 S_{\tau_{n, k}}-\tau_{n, k}\right| \geq x\right] \leq\left(k / p_{n}\right) x^{-2}$.

Hence, for every $n$ and $x$ in $\mathbf{N}, p_{n, k} \leq 4 p_{n} x+\left(k / p_{n}\right) x^{-2}$. Choosing $x=\left\lceil p_{n}^{-2 / 3}\right\rceil$ yields $p_{n, k} \leq 4\left(p_{n}^{1 / 3}+p_{n}\right)+k p_{n}^{1 / 3}$. The result follows.

To finish the proof of theorem 43, we need to remind some standard facts about Vershik's first level criterion, namely definition 2.6, proposition 2.7 and proposition 2.17 of [16].

Let $\mathcal{F}=\left(\mathcal{F}_{n}\right)_{n \leq 0}$ be a filtration with independent increments (Laurent writes that $\mathcal{F}$ is locally of product-type). Given a separable metric space $(E, d)$, one says that a random variable $R \in L^{1}\left(\mathcal{F}_{0}, E\right)$ satisfies Vershik's first level criterion (with respect to $\mathcal{F}$ ) if for every $\delta>0$, one can find an integer $n_{0} \leq 0$, some innovations $V_{n_{0}+1}, \ldots, V_{0}$ of $\mathcal{F}$ at times $n_{0}+1, \ldots, 0$ and some random variable $S \in L^{1}\left(\sigma\left(V_{n_{0}+1}, \ldots, V_{0}\right), E\right)$ such that $\mathbb{E}[d(R, S)]<\delta$.

The subset of random variables in $L^{1}\left(\mathcal{F}_{0}, E\right)$ satisfying Vershik's first level criterion (with respect to $\mathcal{F}$ ) is closed in $L^{1}\left(\mathcal{F}_{0}, E\right)$. If $R \in L^{1}\left(\mathcal{F}_{0}, E\right)$ satisfies Vershik's first level criterion, then any measurable real function of $R$ also satisfies Vershik's first level criterion.

The first step of the proof is to check that for every $m \leq 0$, the random variable $\left(X_{m}(1), \ldots, X_{m}(L)\right)$, taking values in $\{a, b\}^{L}$ endowed with the discrete metric, satisfies Vershik's first level criterion with respect to $\left(\mathcal{F}_{n}^{X, I}\right)_{n \leq 0}$. Indeed, by stationarity, the construction of lemma 47 can be started at any time $n_{0}$ instead of time 0 . Starting this construction at time $n_{0}$ yields innovations $J_{n_{0}+1}^{n_{0}}, J_{n_{0}+2}^{n_{0}}, \ldots$ at times $n_{0}+1, n_{0}+2, \ldots$ Fix two integers $m \leq 0$ and $L \geq 1$. By stationarity, for every $n_{0} \leq m$,

$\mathbb{P}\left[X_{m}=C \circ \psi_{J_{n_{0}+1}^{n_{0}}} \circ \cdots \circ \psi_{J_{m}^{n_{0}}}\right.$ on $\left.\llbracket 1, L \rrbracket\right]=\mathbb{P}\left[X_{m-n_{0}}=C \circ \psi_{J_{1}} \circ \cdots \circ \psi_{J_{m-n_{0}}}\right.$ on $\left.\llbracket 1, L \rrbracket\right]$.

Lemma 49 ensures that this probability tends to 1 as $n \rightarrow+\infty$.

We derive successively that the following random variables also satisfies Vershik's first level criterion:

- $X_{m}$, valued in $\{a, b\}^{\infty}$ endowed with the metric given by

$$
d(x, y)=2^{-\inf \{i \geq 1: x(i) \neq y(i)\}} .
$$


- $\left(X_{m}, I_{m+1}, \ldots, I_{0}\right)$, valued in $\{a, b\}^{\infty} \times \mathcal{P}(\mathbf{N})^{|m|}$ endowed with the product of the metrics defined as above on each factor $\{a, b\}^{\infty}$ or $\mathcal{P}(\mathbf{N})$ identified with $\{0,1\}^{\infty}$;

- any measurable real function of $\left(X_{m}, I_{m+1}, \ldots, I_{0}\right)$;

- any real random variable in $\mathcal{F}_{0}^{X, I}$.

The proof is complete.

\subsection{A non complementable filtration yielding a complementable factor}

We still work with the filtration generated by the uniform randomised decimation process $\left(\left(X_{n}, I_{n}\right)\right)_{n \in \mathbf{Z}}$ on the alphabet $\{a, b\}$. We call $\mathcal{P}^{\prime \prime}(\mathbf{N})$ the set of all infinite subsets of $\mathbf{N}$ with infinite complement, and we set $E=\{a, b\}^{\infty} \times \mathcal{P}^{\prime \prime}(\mathbf{N})$. Since $\nu\left(\mathcal{P}^{\prime \prime}(\mathbf{N})\right)=1$, we may assume and we do assume that the Markov chain $\left(\left(X_{n}, I_{n}\right)\right)_{n \in \mathbf{Z}}$ takes values in $E$.

At each time $n$, we define the random variable $Y_{n}=\psi_{I_{n}^{c}}\left(X_{n-1}\right)$ coding the portion of the infinite word $X_{n-1}$ rejected at time $n$ to get the word $X_{n}$. Of course, the knowledge of $I_{n}, X_{n}$ and $Y_{n}$ enables us to recover $X_{n-1}$ : for every $i \in \mathbf{N}, X_{n-1}(i)$ equals $X_{n}(r)$ or $Y_{n}(r)$ according that $i$ is the $r^{\text {th }}$ element of $I_{n}$ or of $I_{n}^{c}$. We can say more.

Proposition 50. (Properties of the sequences $\left(Y_{n}\right)_{n \in \mathbf{Z}}$ and $\left(I_{n}\right)_{n \in \mathbf{Z}}$ )

1. The random variables $Y_{n}$ are independent and uniform on $\{a, b\}^{\infty}$.

2. The sequence $\left(Y_{n}\right)_{n \in \mathbf{Z}}$ is independent of the sequence $\left(I_{n}\right)_{n \in \mathbf{Z}}$.

3. Each $X_{n}$ is almost surely a measurable function of $I_{n+1}, Y_{n+1}, I_{n+2}, Y_{n+2}, \ldots$.

Note that proposition 50 provides a constructive method to get a uniform randomised decimation process on $\{a, b\}$.

Proof. The first two statements follow from a repeated application of lemma 41, Since the formulas involving the processes $I, X, Y$ are invariant by time-translations, one needs only to check the third statement when $n=0$. For every $i \in \mathbf{N}$, call

$$
N_{i}=\inf \left\{n \geq 1: i \notin \psi_{I_{1}} \circ \cdots \circ \psi_{I_{n}}(\mathbf{N})\right\} .
$$

the first time $n$ at which the letter $X_{0}(i)$ is rejected when forming the word $X_{n}$. For every $n \geq 0,\left[N_{i}>n\right]=\left[i \in \psi_{I_{1}} \circ \cdots \circ \psi_{I_{n}}(\mathbf{N})\right]$; but by lemma 40, the law of the range of $\psi_{I_{1}} \circ \cdots \circ \psi_{I_{n}}$ is $\nu_{2^{-n}}$, so $\mathbb{P}\left[N_{i}>n\right]=2^{-n}$. Therefore, $N_{i}$ is a measurable function of $\left(I_{n}\right)_{n \geq 1}$ and is almost surely finite. On the event $\left[N_{i}<+\infty\right], X_{0}(i)=Y_{N_{i}}\left(R_{i}\right)$, where $R_{i}$ is the rank of $i$ in the set $\psi_{I_{1}} \circ \cdots \circ \psi_{I_{N_{i}}} \circ \psi_{I_{N_{i}+1}^{c}}(\mathbf{N})$. The proof is complete.

We split each random variable $I_{n}$ into two independent random variables, namely $U_{n}=\left\{I_{n}, I_{n}^{c}\right\}$ and $V_{n}=\mathbf{1}_{\left[1 \in I_{n}\right]}$. The random variable $U_{n}$ takes values in the set $\Pi$ of all partitions of $\mathbf{N}$ into two infinite blocks. Given such a partition $u \in \Pi$, we denote by $u(1)$ the block containing 1 and by $u(0)$ its complement. Then $I_{n}=U_{n}\left(V_{n}\right)$ and each one of the random variables $U_{n}, U_{n}(0)$ and $U_{n}(1)$ carries the same information.

Call $\mathcal{C}$ the cylindrical $\sigma$-field on $E^{\mathbf{Z}}$ and $\pi$ the law of $\left(\left(X_{n}, I_{n}\right)\right)_{n \in \mathbf{Z}}$. By stationarity, the shift operator $T$ is an automorphism of $\left(E^{\mathbf{Z}}, \mathcal{C}, \pi\right)$. The formulas defining $U_{n}, V_{n}, Y_{n}$ from $I_{n}$ and $X_{n-1}$ are invariant by time-translations so the measurable maps $\Phi$ and $\Psi$ yielding $\left(U_{n}\right)_{n \in \mathbf{Z}}$ and $\left(\left(V_{n}, Y_{n}\right)\right)_{n \in \mathbf{Z}}$ from $\left(\left(X_{n}, I_{n}\right)\right)_{n \in \mathbf{Z}}$ commute with $T$. Therefore, the $\sigma$-fields $\Phi^{-1}(\mathcal{C})$ and $\Psi^{-1}(\mathcal{C})$ are factors of $T$. 
Theorem 51. 1. The factor $\Phi^{-1}(\mathcal{C})$ is complementable with complement $\Psi^{-1}(\mathcal{C})$. Thus, if the Markov chain $\left(\left(Y_{n}, I_{n}\right)\right)_{n \in \mathbf{Z}}$ is defined on the canonical space $\left(E^{\mathbf{Z}}, \mathcal{C}, \pi\right)$, then $\mathcal{F}_{\infty}^{U}$ is a complementable factor of $T$ with complement $\mathcal{F}_{\infty}^{V, Y}$.

2. Yet, the filtration $\mathcal{F}^{U}$ is not complementable in the filtration $\mathcal{F}^{X, I}$.

Proof. By proposition 50, $\mathcal{F}_{\infty}^{U}$ and $\mathcal{F}_{\infty}^{V, Y}$ are independent, and $\mathcal{F}_{\infty}^{U} \vee \mathcal{F}_{\infty}^{V, Y}=\mathcal{F}_{\infty}^{X, I} \bmod \mathbb{P}$. Therefore, $\Phi^{-1}(\mathcal{C})$ and $\Psi^{-1}(\mathcal{C})$ are independent in $\left(E^{\mathbf{Z}}, \mathcal{C}, \pi\right)$ and $\Phi^{-1}(\mathcal{C}) \vee \Psi^{-1}(\mathcal{C})=\mathcal{C}$ $\bmod \pi$ : the factor $\Phi^{-1}(\mathcal{C})$ is complementable with complement $\Psi^{-1}(\mathcal{C})$.

Let $U=\left(U_{n}\right)_{n \leq 0}$. The random variable $U$ takes values in $\Pi^{\mathbf{Z}_{-}}$. For every $u=$ $\left(u_{n}\right)_{n \leq 0} \in \Pi^{\mathbf{Z}_{-}}$and $n \leq 0$, call $W_{n}^{u}$ the map from $\{0,1\}^{|n|}$ to $\{a, b\}$ defined by

$$
W_{n}^{u}\left(v_{n+1}, \ldots, v_{0}\right)=X_{n} \circ \psi_{u_{n+1}\left(v_{n+1}\right)} \circ \cdots \circ \psi_{u_{0}\left(v_{0}\right)}(1) .
$$

By ordering the elements of $\{0,1\}^{|n|}$ in the lexicographic order, one identifies $W_{n}^{u}$ with an element of $\{a, b\}^{|n|}$.

Since $X_{n}=X_{n-1} \circ \psi_{I_{n}}=X_{n-1} \circ \psi_{u_{n}\left(V_{n}\right)} \mathbb{P}_{u}$-almost surely, we have

$$
W_{n}^{u}\left(v_{n+1}, \ldots, v_{0}\right)=W_{n-1}^{u}\left(V_{n}, v_{n+1}, \ldots, v_{0}\right) \mathbb{P}_{u} \text {-almost surely, }
$$

so $W_{n}^{u}$ is the left half or the right half of $W_{n-1}^{u}$ according $V_{n}$ equals 0 or 1 . Moreover, under $\mathbb{P}_{u}$, the random variable $V_{n}$ is independent of $\mathcal{F}_{n-1}^{W^{u}, V}$ and uniform on $\{0,1\}$.

Hence, under $\mathbb{P}_{u}$, the process $\left(W_{n}^{u}, V_{n}\right)_{n \leq 0}$ is a dyadic split-words process with innovations $\left(V_{n}\right)_{n \leq 0}$. The filtration of this process is known to be non-standard (see [24]). But one checks that $\left(V_{n}\right)_{n \leq 0}$ is also a sequence of innovations of the larger filtration $\left(\mathcal{F}^{X, I}\right)_{n \leq 0}$ seen under $\mathbb{P}_{u}=\mathbb{P}[\cdot \mid U=u]$, so $\left(\mathcal{F}_{n}^{W^{u}, V}\right)_{n \leq 0}$ is immersed in $\left(\mathcal{F}^{X, I}\right)_{n \leq 0}$ and $\left(\mathcal{F}^{X, I}\right)_{n \leq 0}$ is also non-standard under $\mathbb{P}_{u}$.

If $\left(\mathcal{F}_{n}^{U}\right)_{n \leq 0}$ admitted an independent complement $\left(\mathcal{G}_{n}\right)_{n \leq 0}$ in $\left(\mathcal{F}_{n}^{X, I}\right)_{n \leq 0}$, this complement would be immersed in the product-type filtration $\left(\mathcal{F}_{n}^{X, I}\right)_{n \leq 0}$ thus standard. Therefore, for $U(\mathbb{P})$-almost every $u \in \Pi^{\mathbf{Z}_{-}}$, the filtration $\left(\mathcal{F}_{n}^{X, I}\right)_{n \leq 0}$ would be standard under the probability $\mathbb{P}_{u}$, by proposition 0.1 of [18]. This leads to a contradiction.

We are done.

\section{Annex : reminders on partitions and entropy}

We recall here classical definitions and results to make the paper self-contained. Most of them can be found in [23]. See also [22].

In the whole section, we fix a measure-preserving map $T$ from a probability space $(Z, \mathcal{Z}, \pi)$ to itself, whereas $\alpha, \beta, \gamma$ denote measurable countable partitions of $Z$ (here, 'measurable partition' means 'partition into measurable blocks'), and $\mathcal{F}, \mathcal{G}$ denote sub$\sigma$-fields of $\mathcal{Z}$.

We will use the non-negative, continuous and strictly concave function $\varphi:[0,1] \rightarrow \mathbb{R}$ defined by $\varphi(x)=-x \log _{2}(x)$, with the convention $\varphi(0)=0$. The maximum of this function is $\varphi(1 / e)=1 /(e \ln 2)$. 


\subsection{Partitions}

Defining the entropy requires discretizations of the ambient probability space, that is why we introduce countable measurable partitions. Equivalently, we could use discrete random variables. We need a few basic definitions.

Definition 52. One says that $\beta$ is finer than $\alpha$ (and note $\alpha \leq \beta$ ) when each block of $\alpha$ is the union of some collection of blocks of $\beta$, i.e. when $\sigma(\alpha) \subset \sigma(\beta)$.

Definition 53. The (non-empty) intersections $A \cap B$ with $A \in \alpha$ and $B \in \beta$ form a partition; this partition is the coarsest refinement of $\alpha$ and $\beta$ and is denoted by $\alpha \vee \beta$.

Definition 54. More generally, if $\left(\alpha_{k}\right)_{k \in K}$ is a countable family of countable measurable partitions of $Z$, we denote by $\bigvee_{k \in K} \alpha_{k}$ the partition whose blocks are the (non-empty) intersections $\bigcap_{k \in K} A_{k}$ where $A_{k} \in \alpha_{k}$ for every $k \in K$; this partition is the coarsest refinement of the $\left(\alpha_{k}\right)_{k \in K}$; it is still measurable but it can be uncountable.

Definition 55. The partitions $\alpha$ and $\beta$ are independent if and only if $\pi(A \cap B)=$ $\pi(A) \pi(B)$ for every $A \in \alpha$ and $B \in \beta$.

Definition 56. We denote by $T^{-1} \alpha$ the partition defined by

$$
T^{-1} \alpha=\left\{T^{-1}(A): A \in \alpha\right\} .
$$

If $T$ is invertible (i.e. bimeasurable), we denote by $T \alpha$ the partition defined by

$$
T \alpha=\{T(A): A \in \alpha\} .
$$

\subsection{Fischer information and entropy of a partition}

Given $A \in \mathcal{Z}$, we view $-\log _{2} \pi(A)$ as the quantity of information provided by the event $A$ when $A$ occurs, with the convention $-\log _{2} 0=+\infty$. 5 With this definition, the occurence of a rare event provide much information; moreover, the information provided by two independent events $A$ and $B$ occuring at the same time is the sum of the informations provided by each of them separately. The entropy of a countable measurable partition is the mean quantity of information provided by the blocks in it.

Definition 57. The Fischer information of the partition $\alpha$ is the random variable

$$
I_{\alpha}:=\sum_{A \in \alpha}\left(-\log _{2} \pi(A)\right) \mathbf{1}_{A}
$$

The entropy of the partition $\alpha$ is the quantity

$$
H(\alpha)=\mathbb{E}_{\pi}\left[I_{\alpha}\right]=\sum_{A \in \alpha} \varphi(\pi(A)) .
$$

Note that null blocks in $\alpha$ do not give any contribution to the entropy of a partition. Non-trivial partitions have positive entropy. Finite partitions have finite entropy. Infinite countable partition can have finite or infinite entropy.

The previous definition can be generalized as follows.

\footnotetext{
${ }^{5}$ Taking logarithms in base 2 is an arbitrary convention which associates one unity of information to any uniform Bernoulli random variable.
} 
Definition 58. The conditional Fischer information of the partition $\alpha$ with regard to $\mathcal{F}$ is the random variable

$$
I_{\alpha \mid \mathcal{F}}=\sum_{A \in \alpha}\left(-\log _{2} \pi(A \mid \mathcal{F})\right) \mathbf{1}_{A} .
$$

The conditional entropy of the partition $\alpha$ with regard to $\mathcal{F}$ is the quantity

$$
H(\alpha \mid \mathcal{F})=\mathbb{E}_{\pi}\left[I_{\alpha \mid \mathcal{F}}\right] .
$$

Remark 59. By conditional Beppo-Levi theorem,

$$
\mathbb{E}\left[I_{\alpha \mid \mathcal{F}} \mid \mathcal{F}\right]=\sum_{A \in \alpha} \varphi(\pi(A \mid \mathcal{F}))
$$

so

$$
H(\alpha \mid \mathcal{F})=\sum_{A \in \alpha} \mathbb{E}_{\pi}[\varphi(\pi(A \mid \mathcal{F}))]
$$

Given any partition $\eta$ into measurable blocks, we will use the following abbreviated notations: $H(\alpha \mid \eta):=H(\alpha \mid \sigma(\eta)), H(\alpha \mid \eta \vee \mathcal{F}):=H(\alpha \mid \sigma(\eta) \vee \mathcal{F})$.

Note that when $\mathcal{F}$ is the trivial $\sigma$-field $\{\emptyset, Z\}, I_{\alpha \mid \mathcal{F}}$ and $H(\alpha \mid \mathcal{F})$ are equal to $I_{\alpha}$ and $H(\alpha)$.

The following properties are very useful and are checked by direct computation, by using the positivity of Fischer information and the strict concavity of $\varphi$.

Proposition 60. (First properties)

1. $I_{T^{-1} \alpha \mid T^{-1} \mathcal{F}}=I_{\alpha \mid \mathcal{F}} \circ T$ so $H\left(T^{-1} \alpha \mid T^{-1} \mathcal{F}\right)=H(\alpha \mid \mathcal{F})$.

2. $H(\alpha \mid \mathcal{F}) \geq 0$, with equality if and only if $\alpha \subset \mathcal{F} \bmod \pi$.

3. $H(\alpha \mid \mathcal{F}) \leq H(\alpha)$. When $H(\alpha)<+\infty$, equality holds if and only if $\alpha$ is independent of $\mathcal{F}$.

4. If $\mathcal{F} \subset \mathcal{G}$, then $\mathbb{E}\left[I_{\alpha \mid \mathcal{F}} \mid \mathcal{F}\right] \geq \mathbb{E}\left[I_{\alpha \mid \mathcal{G}} \mid \mathcal{F}\right]$ so $H(\alpha \mid \mathcal{F}) \geq H(\alpha \mid \mathcal{G})$.

5. If $\alpha \leq \beta$, then $I_{\alpha \mid \mathcal{F}} \leq I_{\beta \mid \mathcal{F}}$ so $H(\alpha \mid \mathcal{F}) \leq H(\beta \mid \mathcal{F})$.

6. $H(\alpha \vee \beta \mid \mathcal{F})=H(\alpha \mid \mathcal{F})+H(\beta \mid \mathcal{F} \vee \alpha) \leq H(\alpha \mid \mathcal{F})+H(\beta \mid \mathcal{F})$.

The last item above (addition formula above and sub-additivity of entropy) is used repeatedly in the present paper. We will also use the next result.

Proposition 61. (monotone sequence of $\sigma$-fields). Assume that $H(\alpha)<+\infty$.

1. If $\left(\mathcal{F}_{n}\right)_{n \geq 0}$ is a non-decreasing sequence of $\sigma$-fields, then

$$
H\left(\alpha \mid \mathcal{F}_{n}\right) \rightarrow H\left(\alpha \mid \mathcal{F}_{\infty}\right) \text { where } \mathcal{F}_{\infty}=\bigvee_{n \geq 0} \mathcal{F}_{n}
$$

2. If $\left(\mathcal{D}_{n}\right)_{n \geq 0}$ is a non-increasing sequence of $\sigma$-fields, then

$$
H\left(\alpha \mid \mathcal{D}_{n}\right) \rightarrow H\left(\alpha \mid \mathcal{D}_{\infty}\right) \text { where } \mathcal{D}_{\infty}=\bigcap_{n \geq 0} \mathcal{D}_{n}
$$


Proof. Given $A \in \alpha$, the martingale and backward martingale convergence theorems and the continuity of $\varphi$ yield $\varphi\left(\pi\left(A \mid \mathcal{F}_{n}\right)\right) \rightarrow \varphi\left(\pi\left(A \mid \mathcal{F}_{\infty}\right)\right)$ and $\varphi\left(\pi\left(A \mid \mathcal{D}_{n}\right)\right) \rightarrow \varphi\left(\pi\left(A \mid \mathcal{D}_{\infty}\right)\right)$ as $n \rightarrow+\infty$. When the partition $\alpha$ is finite, the convergences $H\left(\alpha \mid \mathcal{F}_{n}\right) \rightarrow H\left(\alpha \mid \mathcal{F}_{\infty}\right)$ and $H\left(\alpha \mid \mathcal{D}_{n}\right) \rightarrow H\left(\alpha \mid \mathcal{D}_{\infty}\right)$ follow by remark 59 ,

The result can be extended to the general case by approximating $\alpha$ with finite mesurable partitions and using the equicontinuity of the maps $\alpha \mapsto \mathbb{E}[\alpha \mid \mathcal{F}]$, where $\mathcal{F}$ is any sub- $\sigma$-field of $\mathcal{Z}$. See propositions 63 and 64 in the next subsection.

\subsection{Continuity properties}

Proposition 62. The formula

$$
d(\alpha, \beta)=H(\alpha \mid \beta)+H(\beta \mid \alpha)=2 H(\alpha \vee \beta)-H(\alpha)-H(\beta)
$$

defines a pseudo-metric on the set of all partitions of $Z$ with finite entropy. Moreover, $d(\alpha, \beta)=0$ if and only if $\sigma(\alpha)=\sigma(\beta)$ modulo $\pi$ (i.e. the non-null blocks of $\alpha$ and $\beta$ are the same modulo $\pi$ ).

Proof. The triangle inequality follows from the inequality

$$
H(\alpha \mid \gamma) \leq H(\alpha \vee \beta \mid \gamma)=H(\beta \mid \gamma)+H(\alpha \mid \beta \vee \gamma) \leq H(\beta \mid \gamma)+H(\alpha \mid \beta)
$$

The other statements follow from proposition 60.

Proposition 63. For the pseudo-metric d thus defined, the set of all finite measurable partitions of $Z$ is dense in the set of all (measurable) partitions on $Z$ with finite entropy.

Proof. Let $\alpha=\left\{A_{n}: n \geq 1\right\}$ be an infinite partition of $Z$ with finite entropy. For every $n \geq 1$, set $\alpha_{n}=\left\{A_{1}, \cdots, A_{n},\left(A_{1} \cup \cdots \cup A_{n}\right)^{c}\right\}$. Since $\alpha$ is finer than $\alpha_{n}$,

$$
H(\alpha) \geq H\left(\alpha_{n}\right) \geq \sum_{k=1}^{n} \varphi\left(\pi\left(A_{k}\right)\right)
$$

so $d\left(\alpha, \alpha_{n}\right)=H(\alpha)-H\left(\alpha_{n}\right) \rightarrow 0$ as $n \rightarrow+\infty$.

Proposition 64. Let $\mathcal{F}$ be a sub- $\sigma$-field of $\mathcal{Z}$. Then, for the pseudo-metric d, the map $\alpha \mapsto H(\alpha \mid \mathcal{F})$ is 1-Lipschitz.

Proof. Let $\alpha$ and $\beta$ be two partitions of $Z$ with finite entropy. Then

$$
H(\beta \mid \mathcal{F})-H(\alpha \mid \mathcal{F}) \leq H(\alpha \vee \beta \mid \mathcal{F})-H(\alpha \mid \mathcal{F})=H(\beta \mid \mathcal{F} \vee \alpha) \leq H(\beta \mid \alpha) \leq d(\alpha, \beta)
$$

The result follows.

Proposition 65. Let $\alpha=\left\{A_{1}, \ldots, A_{n}\right\}$ and $\beta=\left\{B_{1}, \ldots, B_{n}\right\}$ be two finite measurable partitions of $Z$ with the same finite number of blocks. For every $A$ and $B$ in $\mathcal{Z}$, set $\delta(A, B)=\pi(A \triangle B)$. Then

$$
\left.d(\alpha, \beta) \leq \sum_{i=1}^{n} 2 \varphi\left(\delta\left(A_{i}, B_{i}\right) / 2\right)\right)+\sum_{i=1}^{n} \delta\left(A_{i}, B_{i}\right) / \ln 2 .
$$

Therefore, the partition $\alpha$ depends continuously on the blocks $A_{1}, \ldots, A_{n}$. In particular, the map $A \mapsto\left\{A, A^{c}\right\}$ is (uniformly) continuous for the pseudo-metrics $\delta$ and $d$. 
Proof. Fix $i \in \llbracket 1, n \rrbracket$. Then the concavity of $\varphi$ yields

$$
\begin{aligned}
\sum_{j \neq i} \pi\left(B_{j}\right) \varphi\left(\pi\left(A_{i} \mid B_{j}\right)\right) & \leq \pi\left(B_{i}^{c}\right) \varphi\left(\sum_{j \neq i} \frac{\pi\left(B_{j}\right)}{\pi\left(B_{i}^{c}\right)} \pi\left(A_{i} \mid B_{j}\right)\right) \\
& =\pi\left(B_{i}^{c}\right) \varphi\left(\sum_{j \neq i} \frac{\pi\left(A_{i} \cap B_{j}\right)}{\pi\left(B_{i}^{c}\right)}\right) \\
& =\pi\left(B_{i}^{c}\right) \varphi\left(\frac{\pi\left(A_{i} \cap B_{i}^{c}\right)}{\pi\left(B_{i}^{c}\right)}\right) \\
& =\pi\left(A_{i} \cap B_{i}^{c}\right)\left[-\log _{2} \pi\left(A_{i} \cap B_{i}^{c}\right)+\log _{2} \pi\left(B_{i}^{c}\right)\right] \\
& \leq \varphi\left(\pi\left(A_{i} \cap B_{i}^{c}\right)\right) .
\end{aligned}
$$

But the concavity of $\varphi$ also yields $\varphi(x) \leq(1-x) / \ln 2$ for every $x \in[0,1]$, so

$$
\pi\left(B_{i}\right) \varphi\left(\pi\left(A_{i} \mid B_{i}\right)\right) \leq \pi\left(B_{i}\right) \pi\left(A_{i}^{c} \mid B_{i}\right) / \ln 2=\pi\left(A_{i}^{c} \cap B_{i}\right) / \ln 2 .
$$

Hence, by remark 59,

$$
H(\alpha \mid \beta)=\sum_{i, j} \pi\left(B_{j}\right) \varphi\left(\pi\left(A_{i} \mid B_{j}\right)\right) \leq \sum_{i} \varphi\left(\pi\left(A_{i} \cap B_{i}^{c}\right)\right)+\sum_{i} \pi\left(A_{i}^{c} \cap B_{i}\right) / \ln 2 .
$$

A similar upper bound holds for $H(\beta \mid \alpha)$. Summing these two inequalities and using once again the concavity of $\varphi$ yields the statement.

\subsection{Entropy of a measure-preserving map}

First, we define quantities $h(T, \alpha)$.

Proposition 66. (Definition and formula for $h(T, \alpha)$ )

1. The sequence $\left(H_{n}(T, \alpha)\right)_{n \geq 0}$ defined by

$$
H_{n}(T, \alpha)=H\left(\alpha \vee T^{-1} \alpha \vee \cdots \vee T^{-(n-1)} \alpha\right)
$$

is concave. Since $H_{0}(T, \alpha)=0$, the sequence $\left(H_{n}(T, \alpha) / n\right)_{n \geq 1}$ is non-increasing so the limit $h(T, \alpha)=\lim _{n \rightarrow+\infty} H_{n}(T, \alpha) / n$ exists in $[0,+\infty]$.

2. If $H(\alpha)<+\infty$, then $h(T, \alpha)=H\left(\alpha \mid \alpha_{1}^{\infty}\right)$, where $\alpha_{1}^{\infty}=\bigvee_{k \geq 1} T^{-k} \alpha$.

Proof. The first statement follows from the equality

$$
\begin{aligned}
H_{n+1}(T, \alpha)-H_{n}(T, \alpha) & =H\left(\alpha \vee T^{-1} \alpha \vee \cdots \vee T^{-n} \alpha\right)-H\left(T^{-1} \alpha \vee \cdots \vee T^{-n} \alpha\right) \\
& =H\left(\alpha \mid T^{-1} \alpha \vee \cdots \vee T^{-(n-1)} \alpha\right),
\end{aligned}
$$

and the fact that $H(\alpha \mid \mathcal{F})$ is non-increasing with regard to $\mathcal{F}$. Using proposition 61 and Cesàro's lemma yields the second statement.

Definition 67. The entropy of $T$ is

$$
\begin{aligned}
h(T) & =\sup \{h(T, \alpha): \alpha \text { partition of } Z \text { with finite entropy }\} \\
& =\sup \{h(T, \alpha): \alpha \text { finite measurable partition of } Z\} .
\end{aligned}
$$

These two quantities coincide thanks to propositions 63 and 74. 
Proposition 68. For every $r \geq 1, h\left(T^{r}\right)=r h(T)$. If $T$ is also invertible, one has also $h\left(T^{-1}\right)=h(T)$.

Proof. For every $n \geq 1$ and every partition $\alpha$ with finite entropy,

$$
H_{n}\left(T^{r}, \alpha\right) \leq H_{n}\left(T^{r}, \alpha \vee \cdots \vee T^{-(r-1)} \alpha\right)=H_{r n}(T, \alpha)
$$

Dividing by $n$ and letting $n$ go to infinity yields

$$
h\left(T^{r}, \alpha\right) \leq h\left(T^{r}, \alpha \vee \cdots \vee T^{-(r-1)} \alpha\right)=r h(T, \alpha) .
$$

The inequalities $h\left(T^{r}\right) \leq r h(T)$ and $r h(T) \leq h\left(T^{r}\right)$ follow.

If $T$ is invertible, the equalities $\alpha \vee \cdots \vee T^{-(n-1)} \alpha=T^{-(n-1)}\left(\alpha \vee \cdots \vee T^{n-1} \alpha\right)$ follow from proposition 60 item 1 and yield $H_{n}(T, \alpha)=H_{n}\left(T^{-1}, \alpha\right)$, so $h\left(T^{-1}\right)=h(T)$.

\subsection{Generators}

Countable generating partitions help us to compute the entropy of invertible measurepreserving maps.

Definition 69. Assume that $T$ is invertible. A countable measurable partition $\gamma$ is generating (with regard to $T$ ) if the partitions $\left(T^{k} \gamma\right)_{k \in \mathbf{Z}}$ generate $\mathcal{Z}$ modulo the null sets.

Theorem 70. (Kolmogorov - Sinai theorem) If $T$ is invertible and $\gamma$ is a countable generator (with regard to $T$ ), then $h(T)=h(T, \gamma)$.

In the next subsection, we will prove a conditional version of this classical theorem, namely theorem 76 .

Here is the basic example of generator.

Example 71. Let $\Lambda$ be a countable set, $p_{0}:\left(y_{k}\right)_{k \in \mathbf{Z}} \mapsto y_{0}$ the 0 -coordinate projection from $\Lambda^{\mathbf{Z}}$ to $\Lambda, S:\left(y_{k}\right)_{k \in \mathbf{Z}} \mapsto\left(y_{k+1}\right)_{k \in \mathbf{Z}}$ the shift operator on $\Lambda^{\mathbf{Z}}$, and $\mu$ any shiftinvariant probability measure on $\Lambda^{\mathbf{Z}}$. Then the partition $\left\{p_{0}^{-1}\{\lambda\}: \lambda \in \Lambda\right\}$ is generating with regard to $S$.

The interesting fact is that many situations can be reduced to this particular case. The proof of next theorem is outlined in 13 .

Theorem 72. (Rohlin's countable generator theorem) If $(Z, \mathcal{Z}, \pi)$ is a Lebesgue space, $T$ is invertible and aperiodic, i.e. if $\pi\left\{z \in Z: \exists n \geq 1: T^{n}(z)=z\right\}=0$, then $T$ admits a countable generating partition $\gamma=\left\{C_{\lambda}: \lambda \in \Lambda\right\}$. Moreover, the $\gamma$-name map $\Phi$ from $Z$ to $\Lambda^{\mathbf{Z}}$, defined by $\Phi(z)_{k}=\lambda$ whenever $T^{k}(z) \in C_{\lambda}$, is invertible modulo the null sets, when $\Lambda^{\mathbf{Z}}$ is endowed with the probability measure $\Phi(\pi)$. The measure $\Phi(\pi)$ is shift-invariant, so $T$ is isomorphic modulo the null sets to the shift operator on $\Lambda^{\mathbf{Z}}$.

When $T$ is invertible, ergodic and has finite entropy, Krieger's theorem ensures the existence of a finite generator with size at most $\left\lfloor 2^{h(T)}\right\rfloor+1$. We do not use this refinement in the present paper.

Using the remark given in footnote in subsection 2.1. one checks that if $T$ is invertible and $(Z, \mathcal{Z}, \pi)$ is a Lebesgue space, then any factor of $T$ admits a countable generating partition. 


\subsection{Conditional entropy given a factor. Pinsker's formula}

Assume that $T$ is invertible and that $\mathcal{B}$ is a factor of $T$. One may define the entropy of $T$ given $\mathcal{B}$ as follows.

Proposition 73. (Definition and formula for $h(T, \alpha \mid \mathcal{B})$ )

1. The sequence $\left(H_{n}(T, \alpha \mid \mathcal{B})\right)_{n \geq 0}$ defined by

$$
H_{n}(T, \alpha \mid \mathcal{B})=H\left(\alpha \vee T^{-1} \alpha \vee \cdots \vee T^{-(n-1)} \alpha \mid \mathcal{B}\right)
$$

is concave. Since $H_{0}(T, \alpha \mid \mathcal{B})=0$, the sequence $\left(H_{n}(T, \alpha \mid \mathcal{B}) / n\right)_{n \geq 1}$ is non-increasing so the limit $h(T, \alpha \mid \mathcal{B})=\lim _{n \rightarrow+\infty} H_{n}(T, \alpha \mid \mathcal{B}) / n$ exists in $[0,+\infty]$.

2. If $H(\alpha)<+\infty$, then $h(T, \alpha \mid \mathcal{B})=H\left(\alpha \mid \alpha_{1}^{\infty} \vee \mathcal{B}\right)$, where $\alpha_{1}^{\infty}=\bigvee_{k \geq 1} T^{-k} \alpha$ denotes the $\sigma$-field generated by the partitions $\left(T^{-k} \alpha\right)_{k \geq 1}$.

Proof. Since $T^{-1} \mathcal{B}=\mathcal{B}$, one has $H_{n}(T, \alpha \mid \mathcal{B})=H\left(T^{-1} \alpha \vee \cdots \vee T^{-n} \alpha \mid \mathcal{B}\right)$, so

$$
\begin{aligned}
H_{n+1}(T, \alpha \mid \mathcal{B})-H_{n}(T, \alpha \mid \mathcal{B})= & H\left(\alpha \vee T^{-1} \alpha \vee \cdots \vee T^{-n} \alpha \mid \mathcal{B}\right) \\
& -H\left(T^{-1} \alpha \vee \cdots \vee T^{-n} \alpha \mid \mathcal{B}\right) \\
= & H\left(\alpha \mid \sigma\left(T^{-1} \alpha \vee \cdots \vee T^{-(n-1)} \alpha \vee \mathcal{B}\right) .\right.
\end{aligned}
$$

The statements follow, by proposition 61 and Cesàro's lemma.

Proposition 74. Assume that $T$ is invertible and that $\mathcal{B}$ is a factor of $T$. If $\alpha$ and $\gamma$ are two partitions of $Z$ with finite entropy, then $h(T, \alpha \mid \mathcal{B})-h(T, \gamma \mid \mathcal{B}) \leq H(\alpha \mid \gamma) \leq d(\alpha, \gamma)$. Therefore, for the pseudo-metric d, the map $\alpha \mapsto h(T, \alpha \mid \mathcal{B})$ is 1-Lipschitz.

Proof. Set $\alpha_{0}^{n-1}=\alpha \vee T^{-1} \alpha \vee \cdots \vee T^{-(n-1)} \alpha$ and $\gamma_{0}^{n-1}=\gamma \vee T^{-1} \gamma \vee \cdots \vee T^{-(n-1)} \gamma$ for every $n \geq 1$. Then

$$
\begin{aligned}
H\left(\alpha_{0}^{n-1} \mid \mathcal{B}\right)-H\left(\gamma_{0}^{n-1} \mid \mathcal{B}\right) & \leq H\left(\alpha_{0}^{n-1} \vee \gamma_{0}^{n-1} \mid \mathcal{B}\right)-H\left(\gamma_{0}^{n-1} \mid \mathcal{B}\right) \\
& =H\left(\alpha_{0}^{n-1} \mid \mathcal{B} \vee \gamma_{0}^{n-1}\right) \\
& \leq \sum_{k=0}^{n-1} H\left(T^{-k} \alpha \mid \mathcal{B} \vee \gamma_{0}^{n-1}\right) \\
& \leq \sum_{k=0}^{n-1} H\left(T^{-k} \alpha \mid T^{-k} \gamma\right) \\
& =n H(\alpha \mid \gamma) .
\end{aligned}
$$

Dividing by $n$ and letting $n$ go to infinity yields $h(T, \alpha \mid \mathcal{B})-h(T, \gamma \mid \mathcal{B}) \leq H(\alpha \mid \gamma) \leq d(\alpha, \gamma)$. The result follows.

Definition 75. The conditional entropy of $T$ given $\mathcal{B}$ is the quantity

$$
\begin{aligned}
h(T \mid \mathcal{B}) & =\sup \{h(T, \alpha \mid \mathcal{B}): \alpha \text { partition of } Z \text { with finite entropy }\} \\
& =\sup \{h(T, \alpha \mid \mathcal{B}): \alpha \text { finite measurable partition of } Z\} .
\end{aligned}
$$

These two quantities coincide thanks to propositions 63 and 74.

Kolmogorov - Sinai theorem admits the following generalization. 
Theorem 76. If $\gamma$ is a countable generator of $T$, then $h(T \mid \mathcal{B})=h(T, \gamma \mid \mathcal{B})$.

Proof. For every integers $p \leq q$, set

$$
\gamma_{p}^{q}=\bigvee_{k=p}^{q} T^{-k} \gamma
$$

Fix $r \geq 0$. Then for every integer $n \geq 1$,

$$
\frac{1}{n} H_{n}\left(T, \gamma_{-r}^{r} \mid \mathcal{B}\right)=\frac{1}{n} H\left(T, \gamma_{-r-n+1}^{r} \mid \mathcal{B}\right)=\frac{n+2 r}{n} \times \frac{1}{n+2 r} H\left(T, \gamma_{-r-n+1}^{r} \mid \mathcal{B}\right) .
$$

Letting $n$ go to infinity yields $h\left(T, \gamma_{-r}^{r} \mid \mathcal{B}\right)=h(T, \gamma \mid \mathcal{B})$. Thus, applying proposition 74 any partition $\alpha$ of $Z$ with finite entropy and yo $\gamma_{-r}^{r}$ yields

$$
h(T, \alpha \mid \mathcal{B})-h(T, \gamma \mid \mathcal{B}) \leq H\left(\alpha \mid \gamma_{-r}^{r}\right) .
$$

But $H\left(\alpha \mid \gamma_{-r}^{r}\right) \rightarrow H(\alpha \mid \mathcal{Z})=0$ as $r \rightarrow+\infty$ since $\gamma$ is a countable generator of $T$. Hence $h(T, \alpha \mid \mathcal{B}) \leq h(T, \gamma \mid \mathcal{B})$. The conclusion follows.

Proposition 77. (Pinsker's formula) Assume that $\alpha$ and $\beta$ have finite entropy. Let $\mathcal{A}$ and $\mathcal{B}$ be two factors generated by $\alpha$ and $\beta$. Set $\alpha_{1}^{\infty}=\bigvee_{k \geq 1} T^{-k} \alpha$ and $\beta_{1}^{\infty}=\bigvee_{k \geq 1} T^{-k} \beta$. Then

$$
h((T, \mathcal{A}) \mid \mathcal{B})=h(T, \mathcal{A} \vee \mathcal{B})-h(T, \mathcal{B})
$$

or equivalently,

$$
H\left(\alpha \mid \alpha_{1}^{\infty} \vee \mathcal{B}\right)=H\left(\alpha \vee \beta \mid \alpha_{1}^{\infty} \vee \beta_{1}^{\infty}\right)-H\left(\beta \mid \beta_{1}^{\infty}\right)
$$

Proof. For every integers $p \leq q$, define the partitions $\alpha_{p}^{q}$ and $\beta_{p}^{q}$ like in the proof above. Then for every non-negative integer $n$,

$$
\begin{aligned}
H_{n+1}(T, \alpha \vee \beta)-H_{n+1}(T, \beta) & =H\left(\alpha_{-n}^{0} \vee \beta_{-n}^{0}\right)-H\left(\beta_{-n}^{0}\right) \\
& =\sum_{k=0}^{n} H\left(T^{k} \alpha \mid \alpha_{-(k-1)}^{0} \vee \beta_{-n}^{0}\right) \\
& =\sum_{k=0}^{n} H\left(\alpha \mid \alpha_{1}^{k} \vee \beta_{k-n}^{k}\right) .
\end{aligned}
$$

By proposition 61, $H\left(\alpha \mid \alpha_{1}^{k} \vee \beta_{-\ell}^{k}\right) \rightarrow H\left(\alpha \mid \alpha_{1}^{\infty} \vee \mathcal{B}\right)$ as $k \rightarrow+\infty$ and $\ell \rightarrow+\infty$. Since the quantities $H\left(\alpha \mid \alpha_{1}^{k} \vee \beta_{k-n}^{k}\right)$ belong to the finite interval $[0, H(\alpha)]$, one deduces that

$$
h(T, \alpha \vee \beta)-h(T, \beta)=\lim _{n \rightarrow+\infty} \frac{1}{n+1}\left(H_{n+1}(T, \alpha \vee \beta)-H_{n+1}(T, \beta)\right)=H\left(\alpha \mid \alpha_{1}^{\infty} \vee \mathcal{B}\right)
$$

Hence, the statement follows from proposition [66, theorem [70, proposition 73 and theorem 76 ,

\section{Acknowledgements}

I thank A. Coquio, J. Brossard, M. Émery, S. Laurent, J.P. Thouvenot for their useful remarks and for stimulating conversations. 


\section{References}

[1] S. Attal, K. Burdzy, M. Émery, Y. Hu, Sur quelques filtrations et transformations browniennes, Séminaire de Probabilités, IXXX, Lecture Notes in Mathematics 1613, 56-69 (1995).

[2] M.T. Barlow, M. Émery, F.B. Knight, S. Song, M. Yor, Autour d'un théorème de Tsirelson sur des filtrations browniennes et non browniennes, Séminaire de Probabilités, XXXII, Lecture Notes in Mathematics 1686, 264-305 (1998).

[3] Berg K., Convolution of invariant measures, maximal entropy, Mathematical Systems Theory 3-2,146-150 (1969).

[4] J. Brossard, C. Leuridan, Filtrations browniennes et compléments indépendants, Séminaire de Probabilités, XLI, Lecture Notes in Mathematics 1934, 265-278 (2008).

[5] J. Brossard, M. Émery, C. Leuridan, Maximal Brownian motions, Annales de l'IHP 45-3, 876-886 (2009).

[6] J. Brossard, M. Émery, C. Leuridan, Skew-Product Decomposition of Planar Brownian Motion and Complementability, Séminaire de Probabilités, XLVI, Lecture Notes in Mathematics 2123, 377-394 (2014).

[7] G. Ceillier, The filtration of the split-words process. Probability Theory and Related Fields, 153, no 1-2, 269-292 (2012).

[8] G. Ceillier, C. Leuridan, Filtrations at the threshold of standardness. Probability Theory and Related Fields, 158, no 3-4, 785-808 (2014).

[9] I. Cornfeld, S. Fomin, Y. Sinai, Ergodic Theory, Grundlehren der mathematischen Wissenschaften Volume 245, Springer (1982).

[10] T. de la Rue, Espaces de Lebesgue. Séminaire de Probabilités XXVII, Lecture Notes in Mathematics 1557, 15-21 (1993).

[11] M. Émery, On certain almost Brownian filtrations. Annales de l'IHP, Probabilités et Statistiques41, 285-305 (2005).

[12] M. Émery, W. Schachermayer, On Vershik's standardness criterion and Tsirelson's notion of cosiness. Séminaire de Probabilités, XXXV, Lecture Notes in Mathematics 1755, 265-305 (2001).

[13] Kalikow S., McCutcheon R., An Outline of Ergodic theory, Cambridge studies in advanced mathematics (2010).

[14] S. Laurent, Filtrations à temps discret négatif, $\mathrm{PhD}$ thesis, Université Louis Pasteur, Institut de Recherche en Mathématique Avancée, Strasbourg (2004).

[15] S. Laurent, On Vershikian and I-cosy random variables and filtrations. (Russian summary) Teor. Veroyatn. Primen. 55, no. 1, 104-132 (2010); translation in Theory Probab. Appl. 55, no. 1, 54-76 (2011).

[16] S. Laurent, On standardness and I-cosiness. Séminaire de Probabilités, XLIII, Lecture Notes in Mathematics 2006, 127-186 (2011). 
[17] S. Laurent, The filtration of erased-word processes. Séminaire de Probabilités, XLVIII, Lecture Notes in Mathematics 2168, 445-458 (2017).

[18] C. Leuridan, Poly-adic Filtrations, standardness, complementability and maximality, Annals of Probability, 45-2, 1218-1246 (2017).

[19] D. Ornstein, Factors of Bernoulli shifts are Beroulli shifts, Advances in Mathematics, 5, 349-364 (1971).

[20] D. Ornstein, Factors of Bernoulli shifts, Israel Journal of Mathematics, 21-2-3, 145-153 (1975).

[21] D. Ornstein, B. Weiss, Finitely determined implies very weak Bernoulli, Israel Journal of Mathematics, 17-1, 94-104 (1974).

[22] W. Parry, Entropy and Generators in Ergodic Theory, W.A. Benjamin, Inc. (1969).

[23] K. Petersen, Ergodic Theory, Cambridge University Press. (1981).

[24] M. Smorodinsky, Processes with no standard extension. Israel Journal of Mathematics, 107, 327-331 (1998).

[25] J.P. Thouvenot, Une classe de systèmes pour lesquels la conjecture de Pinsker est vraie Israel Journal of Mathematics, 21, 208-214 (1975).

[26] M. Tsirelson, About Yor's problem, avalaible on http://www.tau.ac.il/ tsirel/download/yor3.pdf (unpublished notes).

[27] A. Vershik, Theory of decreasing sequences of measurable partitions. Algebra i Analiz, 6:4 (1994), 1-68. English Tranlation: St. Petersburg Mathematical Journal, 6:4 (1995), 705-761.

[28] H. von Weizsäcker, Exchanging the order of taking suprema and countable intersections of $\sigma$-algebras, Ann. Inst. H. Poincaré Sect. B, 19, no. 1, 91-100 (1983). 